\title{
Traces on ideals in pivotal categories
}

\author{
Nathan Geer, Bertrand Patureau-Mirand and Alexis Virelizier ${ }^{1}$
}

\begin{abstract}
We construct invariants of $\mathcal{C}$-colored spherical graphs from traces on ideals in a pivotal category $\ell$. Then we provide a systematic approach to defining such traces from ambidextrous and spherical traces on a class of objects of $\ell$. This extends the notion of an ambidextrous object of a braided category given by the first two authors in a previous work.
\end{abstract}

Mathematics Subject Classification (2010). Primary 18D10; Secondary $16 \mathrm{~T} 05$.

Keywords. Pivotal category, trace, ideal, ambidextrous.

\section{Contents}

1 Pivotal categories . . . . . . . . . . . . . . . . . 93

2 Traces on ideals . . . . . . . . . . . . . . . . . . 97

3 Invariants of closed graphs . . . . . . . . . . . . . . . 102

4 Traces and ideals from classes of objects . . . . . . . . . . . . . . 109

5 The case of projectives and the slope . . . . . . . . . . . . 120

References . . . . . . . . . . . . . . . . . . . . . 123

\section{Introduction}

Many constructions of quantum topology rely on tensor categories with duality and more precisely on categorical traces of such categories. When these categories are non-semisimple, the categorical traces are often degenerate and these constructions become trivial. In [3] and [7] it has been shown that in non-semisimple ribbon categories, non-trivial traces can exist. The study of these traces leads to new interesting quantum invariants of links and 3-manifolds. The goal of this paper is to generalize this study within the context of pivotal categories. In particular, we develop a theory of modified traces on ideals in pivotal categories and show that such traces lead to topological invariants of planar graphs.

\footnotetext{
${ }^{1}$ The work of Nathan Geer has been partially supported by the NSF grants DMS-0706725 and DMS0968279. Alexis Virelizier was partially supported by the ANR grant GESAQ.
} 
Let $\mathcal{C}$ be a pivotal $\mathbb{k}$-category. We call a class $\mathcal{I}$ of objects of $\mathcal{C}$ a left ideal (resp. a right ideal) if it is closed under retraction and left (resp. right) tensor multiplication by objects of $\mathcal{C}$. An ideal is a two-side ideal. A left trace (resp. a right trace) on a left (resp. right) ideal $\mathcal{I}$ is a family of $\mathbb{k}$-linear functions

$$
\mathrm{t}=\left\{\mathrm{t}_{V}: \operatorname{End}_{e}(V) \rightarrow \mathbb{k}\right\}_{V \in \mathcal{I}}
$$

which is suitably compatible with the tensor product and composition of morphisms (cf. Section 2.2). A trace on an ideal $\mathcal{I}$ is a family $t=\left\{\mathrm{t}_{V}\right\}_{V \in \mathcal{I}}$ which is both a left and right trace on $\mathcal{I}$.

Let us explain how left traces and traces lead to topological invariants of certain graphs. Similar invariants exist for right traces. We consider planar and spherical graphs (i.e., graphs in $\mathbb{R}^{2}$ and $S^{2}=\mathbb{R}^{2} \cup\{\infty\}$, respectively). If $\mathcal{O}$ is a class of objects of $\mathcal{C}$, then a $\mathcal{O}$-colored graph in $\mathbb{R}^{2}$ (resp. $S^{2}$ ) is a ribbon graph embedded in $\mathbb{R}^{2}$ (resp. $S^{2}$ ) whose edges are colored by elements of $\mathcal{O}$ and coupons are colored by morphisms in $\ell$. Let $\Gamma$ be a planar $\ell$-colored graph. By a left cutting presentation

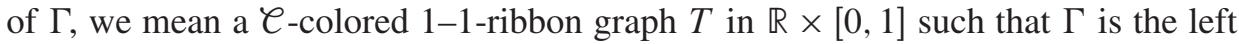
closure of $T$. Let $t$ be a left trace on a left ideal $\mathcal{I}$ of $\mathcal{C}$. We say $\Gamma$ is $\mathcal{I}$-admissible if it admits a left cutting presentation $T$ whose open component is colored by an object $V$ of $\mathcal{I}$. For such a left cutting presentation we set

$$
F_{\mathrm{t}}(\Gamma)=\mathrm{t}_{V}\left(f_{T}\right)
$$

where $f_{T}: V \rightarrow V$ is the morphism defined by $T$ via Penrose calculus (see Subsection 1.3). In Section 3 we prove the following statements.

(i) Let $\Gamma$ be an $\mathcal{I}$-admissible planar graph. Then the scalar $F_{t}(\Gamma)$ is well defined (that is, independent of the choice of the left cutting presentation $T$ of $\Gamma$ ). The assignment $\Gamma \mapsto F_{\mathrm{t}}(\Gamma)$ is then an isotopy invariant of $\mathcal{I}$-admissible planar graphs $\Gamma$.

(ii) The left trace t on the left ideal $\mathcal{I}$ determines a class $A \subset \mathcal{I}$ (see (9)) such that for any A-colored spherical graph $\Gamma$ the scalar $F_{\mathrm{t}}(\Gamma)$ is well defined. The assignment $\Gamma \mapsto F_{\mathrm{t}}(\Gamma)$ is then an isotopy invariant of A-colored spherical graphs $\Gamma$.

(iii) If $\mathcal{I}$ is an ideal and t is a trace on $\mathcal{I}$, then $\mathrm{A}=\mathcal{I}$ and so the assignment $\Gamma \mapsto F_{\mathrm{t}}(\Gamma)$ is a well defined isotopy invariant of $A$-colored spherical graphs $\Gamma$. Moreover, this assignment extends to an isotopy invariant of $\mathcal{I}$-admissible spherical graphs $\Gamma$.

In Section 4 we give a systematic approach to defining traces on ideals. More precisely, let $\mathcal{O}$ be a class of objects of $\mathcal{C}$. We define a left ambidextrous trace on $\mathcal{O}$ as a family $t=\left\{t_{X}: \operatorname{End} \mathcal{C}(X) \rightarrow \mathbb{k}\right\}_{X \in \mathcal{O}}$ of $\mathbb{k}$-linear forms satisfying

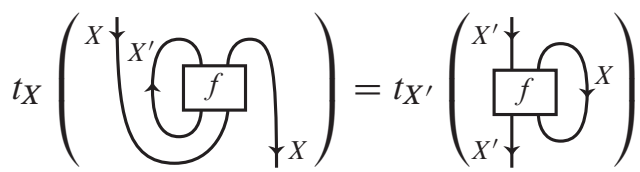


for all $X, X^{\prime} \in \mathcal{O}$ and $f \in \operatorname{End} \varphi\left(X^{\prime} \otimes X^{*}\right)$. Then we show that left ambidextrous traces on $\mathcal{O}$ bijectively correspond to left traces on the left ideal generated by $\mathcal{O}$. We also give a notion of a spherical trace on $\mathcal{O}$ and show that these traces bijectively correspond to traces on the ideal generated by $\mathcal{O}$.

The invariant of spherical graphs described above in (ii) relies on a certain set $\mathrm{A}$ of objects defined from a one-sided trace on a one-sided ideal. In Section 5 we give a characterization of $A$, in terms of the slope, when the one-sided ideal is the ideal of projective objects. In [5], the slope and left ambidextrous traces are used to construct 3-manifold invariants from the categories of finite dimensional modules over the nonrestricted quantum groups associated to simple Lie algebras introduced and studied by De Concini, Kac, Procesi, Reshetikhin, and Rosso in [1] and [2]. The categories of finite dimensional representations of these quantum groups cannot be braided as their tensor product is not commutative up to isomorphism.

In [4] the results of this paper are used to prove that traces on the ideal of projective modules exist for factorizable ribbon Hopf algebras, modular representations of finite groups and their quantum doubles, complex and modular Lie (super)algebras, the $(1, p)$ minimal model in conformal field theory, and quantum groups at a root of unity. In all these examples the usual trace restricted to the ideal of projective modules is zero but the modified trace suggested in this paper is non-zero.

Throughout the paper, we fix a commutative ring $\mathbb{k}$.

\section{Pivotal categories}

In this section we recall some well-known properties concerning pivotal categories. Given a category $\mathcal{C}$, the notation $X \in \mathcal{C}$ means that $X$ is an object of $\mathcal{C}$.

1.1. Pivotal categories. Recall that a pivotal (or sovereign) category is a (strict) monoidal category $\mathcal{C}$, with unit object $\mathbb{1}$, such that to each object $X \in \mathcal{C}$ there are associated a dual object $X^{*} \in \mathcal{C}$ and four morphisms

$$
\begin{aligned}
& \operatorname{ev}_{X}: X^{*} \otimes X \longrightarrow \mathbb{1}, \quad \operatorname{coev}_{X}: \mathbb{1} \longrightarrow X \otimes X^{*}, \\
& \widetilde{\operatorname{ev}}_{X}: X \otimes X^{*} \longrightarrow \mathbb{1}, \quad \widetilde{\operatorname{cov}_{X}: \mathbb{1} \longrightarrow X^{*} \otimes X,}
\end{aligned}
$$

such that $\left(\mathrm{ev}_{X}, \operatorname{coev}_{X}\right)$ is a left duality for $X,\left(\widetilde{\mathrm{ev}}_{X}, \widetilde{\operatorname{cov}}_{X}\right)$ is a right duality for $X$, and the induced left and right dual functors coincide as monoidal functors; see [8]. In particular, the left dual and right dual of a morphism $f: X \rightarrow Y$ in $\mathcal{C}$ coincide,

$$
\begin{aligned}
f^{*} & =\left(\operatorname{ev}_{Y} \otimes \operatorname{id}_{X^{*}}\right)\left(\operatorname{id}_{Y^{*}} \otimes f \otimes \operatorname{id}_{X^{*}}\right)\left(\operatorname{id}_{Y^{*}} \otimes \operatorname{coev}_{X}\right) \\
& =\left(\operatorname{id}_{X^{*}} \otimes \widetilde{\operatorname{ev}}_{Y}\right)\left(\operatorname{id}_{X^{*}} \otimes f \otimes \operatorname{id}_{Y^{*}}\right)\left(\widetilde{\operatorname{cov}_{X}} \otimes \operatorname{id}_{Y^{*}}\right): Y^{*} \longrightarrow X^{*},
\end{aligned}
$$

and

$$
\varphi=\left\{\varphi_{X}=\left(\widetilde{\mathrm{ev}}_{X} \otimes \mathrm{id}_{X^{* *}}\right)\left(\mathrm{id}_{X} \otimes \operatorname{coev}_{X^{*}}\right): X \longrightarrow X^{* *}\right\}_{X \in \mathcal{C}}
$$


is a monoidal natural isomorphism, called the pivotal structure.

1.2. Traces and dimensions. Let $\ell$ be pivotal category. Recall that Ende $(\mathbb{1})$ is a commutative monoid. The left trace $\operatorname{tr}_{l}(f) \in \operatorname{End}_{e}(\mathbb{1})$ and the right trace $\operatorname{tr}_{r}(f) \in$ Ende $(\mathbb{1})$ of an endomorphism $f$ of an object $X$ of $C$ are defined by

$$
\left\{\begin{array}{l}
\operatorname{tr}_{l}(f)=\operatorname{ev}_{X}\left(\operatorname{id}_{X} * \otimes f\right) \widetilde{\operatorname{coev}} X \\
\operatorname{tr}_{r}(f)=\widetilde{\operatorname{ev}}_{X}\left(f \otimes \operatorname{id}_{X} *\right) \operatorname{coev}_{X}
\end{array}\right.
$$

Both traces are symmetric: $\operatorname{tr}_{l}(g h)=\operatorname{tr}_{l}(h g)$ and $\operatorname{tr}_{r}(g h)=\operatorname{tr}_{r}(h g)$ for any mor-

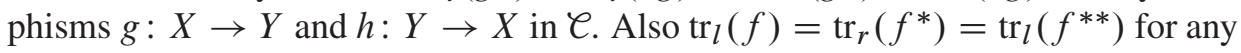
endomorphism $f$ of an object (and similarly with $l, r$ exchanged). If

$$
\alpha \otimes \operatorname{id}_{X}=\operatorname{id}_{X} \otimes \alpha \quad \text { for all } \alpha \in \operatorname{End}_{\ell}(\mathbb{1}) \text { and } X \in \mathcal{C},
$$

then the traces $\operatorname{tr}_{l}, \operatorname{tr}_{r}$ are $\otimes$-multiplicative, that is

$$
\left\{\begin{array}{l}
\operatorname{tr}_{l}(f \otimes g)=\operatorname{tr}_{l}(f) \operatorname{tr}_{l}(g), \\
\operatorname{tr}_{r}(f \otimes g)=\operatorname{tr}_{r}(f) \operatorname{tr}_{r}(g),
\end{array}\right.
$$

for all endomorphisms $f, g$ of objects of $\ell$.

The left and right dimensions of $X \in \mathrm{Ob}(\mathcal{C})$ are defined by $\operatorname{dim}_{l}(X)=\operatorname{tr}_{l}\left(\mathrm{id}_{X}\right)$ and $\operatorname{dim}_{r}(X)=\operatorname{tr}_{r}\left(\operatorname{id}_{X}\right)$. Clearly, $\operatorname{dim}_{l}(X)=\operatorname{dim}_{r}\left(X^{*}\right)=\operatorname{dim}_{l}\left(X^{* *}\right)$ (and similarly with $l, r$ exchanged). Note that isomorphic objects have the same dimensions and $\operatorname{dim}_{l}(\mathbb{1})=\operatorname{dim}_{r}(\mathbb{1})=\mathrm{id}_{\mathbb{1}}$. If the category $\zeta$ satisfies (1), then left and right dimensions are $\otimes$-multiplicative: $\operatorname{dim}_{l}(X \otimes Y)=\operatorname{dim}_{l}(X) \operatorname{dim}_{l}(Y)$ and $\operatorname{dim}_{r}(X \otimes Y)=\operatorname{dim}_{r}(X) \operatorname{dim}_{r}(Y)$ for any $X, Y \in \mathcal{C}$.

1.3. Penrose graphical calculus. We represent morphisms in a category $\ell$ by plane diagrams to be read from the bottom to the top. The diagrams are made of oriented arcs colored by objects of $\ell$ and of boxes (called coupons) colored by morphisms of $\ell$. The arcs connect the boxes and have no mutual intersections or self-intersections. The identity $\operatorname{id}_{X}$ of $X \in \mathcal{C}$, a morphism $f: X \rightarrow Y$, and the composition of two morphisms $f: X \rightarrow Y$ and $g: Y \rightarrow Z$ are represented as

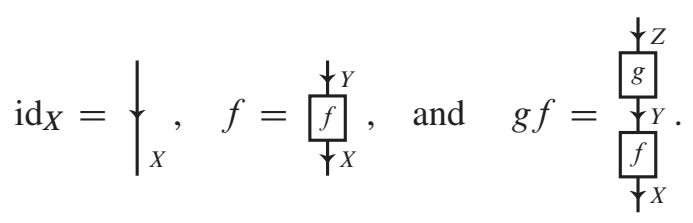

If $\varphi$ is monoidal, then the monoidal product of two morphisms $f: X \rightarrow Y$ and $g: U \rightarrow V$ is represented by juxtaposition 


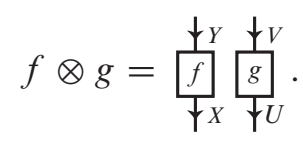

In a pivotal category, if an arc colored by $X$ is oriented upwards, then the corresponding object in the source/target of morphisms is $X^{*}$. For example, $\mathrm{id}_{X^{*}}$ and a morphism $f: X^{*} \otimes Y \rightarrow U \otimes V^{*} \otimes W$ may be depicted as

$$
\operatorname{id}_{X *}=\uparrow_{X}=\downarrow_{X^{*}}
$$

and

$$
f=\frac{\downarrow_{U}+V \nmid W}{\uparrow_{X} \downarrow_{Y}}
$$

The duality morphisms are depicted as

$$
\begin{aligned}
& \mathrm{ev}_{X}=\bigcap_{x}, \quad \operatorname{coev}_{X}=\bigcup_{X}, \\
& \widetilde{\mathrm{ev}}_{X}=\bigcap_{X}, \quad \widetilde{\operatorname{cov}}_{X}=\bigcup_{X} .
\end{aligned}
$$

The dual of a morphism $f: X \rightarrow Y$ and the traces of a morphism $g: X \rightarrow X$ can be depicted as

$$
\left.f^{*}=\{f\}^{x}={ }^{x} \uparrow f\right\}_{Y}
$$

and

$$
\left\{\begin{array}{l}
\operatorname{tr}_{l}(g)=x \bigcirc g, \\
\operatorname{tr}_{r}(g)=g f^{x} .
\end{array}\right.
$$

If $\mathcal{C}$ is pivotal, then the morphisms represented by the diagrams are invariant under isotopies of the diagrams in the plane keeping fixed the bottom and top endpoints.

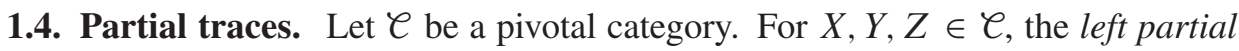
trace (with respect to $X$ ) is the map

$$
\operatorname{tr}_{l}^{X}: \operatorname{Home}_{e}(X \otimes Y, X \otimes Z) \longrightarrow \operatorname{Home}_{e}(Y, Z)
$$

defined for $f \in \operatorname{Home}_{e}(X \otimes Y, X \otimes Z)$ by

$$
\operatorname{tr}_{l}^{X}(f)=\left(\operatorname{ev}_{X} \otimes \operatorname{id}_{Z}\right)\left(\operatorname{id}_{X^{*}} \otimes f\right)\left(\widetilde{\operatorname{cov}}_{X} \otimes \operatorname{id}_{Y}\right)=X \widehat{\underbrace{+Z}_{f}} .
$$


Likewise, the right partial trace (with respect to $X$ ) is the map

$$
\operatorname{tr}_{r}^{X}: \operatorname{Home}_{e}(Y \otimes X, Z \otimes X) \longrightarrow \operatorname{Hom}_{e}(Y, Z)
$$

defined, for $g \in \operatorname{Home}_{e}(Y \otimes X, Z \otimes X)$ by

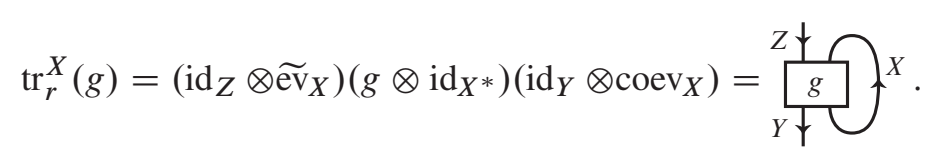

Note that $\operatorname{tr}_{r}^{X^{*}}\left(f^{*}\right)=\left(\operatorname{tr}_{l}^{X}(f)\right)^{*}$ and $\operatorname{tr}_{l}^{X^{*}}\left(g^{*}\right)=\left(\operatorname{tr}_{r}^{X}(g)\right)^{*}$. In particular

$$
\operatorname{tr}_{l}^{X^{* *}}\left(f^{* *}\right)=\left(\operatorname{tr}_{l}^{X}(f)\right)^{* *}=\varphi_{Y} \operatorname{tr}_{l}^{X}(f) \varphi_{Y}^{-1}
$$

and

$$
\operatorname{tr}_{r}^{X^{* *}}\left(g^{* *}\right)=\left(\operatorname{tr}_{r}^{X}(g)\right)^{* *}=\varphi_{X} \operatorname{tr}_{r}^{X}(g) \varphi_{X}^{-1},
$$

where $\varphi=\left\{\varphi_{X}: X \rightarrow X^{* *}\right\}_{X \in \mathcal{C}}$ is the pivotal structure of $\mathcal{C}$.

Note that if $f: X \rightarrow X$ is an endomorphism in $\ell$, then

$$
\begin{aligned}
& \operatorname{tr}_{l}^{X}(f)=\operatorname{tr}_{l}(f), \\
& \operatorname{tr}_{r}^{X}(f)=\operatorname{tr}_{r}(f),
\end{aligned}
$$

and

$$
\operatorname{tr}_{l}^{\mathbb{1}}(f)=f=\operatorname{tr}_{r}^{\mathbb{1}}(f)
$$

1.5. Spherical categories. A spherical category is a pivotal category whose left and right traces are equal, i.e., $\operatorname{tr}_{l}(f)=\operatorname{tr}_{r}(f)$ for every endomorphism $f$ of an object. Then $\operatorname{tr}_{l}(f)$ and $\operatorname{tr}_{r}(f)$ are denoted $\operatorname{tr}(f)$ and called the trace of $f$. Similarly, the left and right dimensions of an object $X$ are equal, denoted $\operatorname{dim}(X)$, and called the dimension of $X$.

For spherical categories, the corresponding Penrose graphical calculus has the following property: the morphisms represented by diagrams are invariant under isotopies of diagrams in the 2-sphere $S^{2}=\mathbb{R}^{2} \cup\{\infty\}$, i.e., are preserved under isotopies pushing arcs of the diagrams across $\infty$. For example, the diagrams above representing $\operatorname{tr}_{l}(f)$ and $\operatorname{tr}_{r}(f)$ are related by such an isotopy. The condition $\operatorname{tr}_{l}(f)=\operatorname{tr}_{r}(f)$ for all $f$ is therefore necessary (and in fact sufficient) to ensure this property.

1.6. Linear categories. A monoidal $\mathbb{k}$-category is a monoidal category $C$ such that its hom-sets are (left) $\mathbb{k}$-modules, the composition and monoidal product of morphisms are $\mathbb{k}$-bilinear, and Ende $(\mathbb{1})$ is a free $\mathbb{k}$-module of rank one. Then the map $\mathbb{k} \rightarrow$ Ende $_{e}(\mathbb{1}), k \mapsto k \mathrm{id}_{\mathbb{1}}$ is a $\mathbb{k}$-algebra isomorphism. It is used to identify $\operatorname{End}_{e}(\mathbb{1})=\mathbb{k}$.

A pivotal $\mathbb{k}$-category satisfies (1). Therefore, the traces $\operatorname{tr}_{l}, \operatorname{tr}_{r}$ and the dimensions $\operatorname{dim}_{l}, \operatorname{dim}_{r}$ in such a category are $\otimes$-multiplicative. Clearly, $\operatorname{tr}_{l}, \operatorname{tr}_{r}$ are $\mathbb{k}$-linear. 
An object $X$ of a monoidal $\mathbb{k}$-category $\mathcal{C}$ is simple if $\operatorname{End} \mathcal{C}(X)$ is a free $\mathbb{k}$-module of rank 1. Equivalently, $X$ is simple if the $\mathbb{k}$-homomorphism $\mathbb{k} \rightarrow \operatorname{End}_{\bigodot}(X), k \mapsto k \operatorname{id}_{X}$ is an isomorphism. By the definition of a monoidal $\mathbb{k}$-category, the unit object $\mathbb{1}$ is simple. If $X$ is a simple object of $\mathcal{C}$, we denote by \langle\rangle$_{V}: \operatorname{End}_{\mathcal{C}}(V) \rightarrow \mathbb{k}$ the inverse of the $\mathbb{k}$-linear isomorphism $\mathbb{k} \rightarrow$ Ende $_{\ell}(V)$ defined by $k \mapsto k \mathrm{id}_{V}$.

\section{Traces on ideals}

In this section we introduce the notion of one-side ideals and one-side traces on one-side ideals. We also study some basic properties of such ideals and traces.

The notation $\mathcal{O} \subset \mathcal{C}$ means that $\mathcal{O}$ is a class of objects of $\mathcal{C}$. If $\mathcal{C}$ is a monoidal category, we denote by $\ell^{\text {rev }}$ the category $\zeta$ with opposite monoidal product defined by $X \otimes{ }^{\text {op }} Y=Y \otimes X$ for $X, Y \in \mathcal{C}$.

2.1. Ideals. By a retract of an object $X$ of a category $\mathcal{C}$, we mean an object $U$ of $\mathcal{C}$ such that there exist morphisms $p: X \rightarrow U$ and $q: U \rightarrow X$ verifying $p q=\mathrm{id}_{U}$.

A class $\mathcal{O} \subset \mathcal{C}$ is said to be closed under retraction if any retract (in $\mathcal{C}$ ) of an object of $\mathcal{O}$ belongs to $\mathcal{O}$.

A class $\mathcal{O}$ of objects of a monoidal category $\mathcal{C}$ is said to be closed under left (resp. right) multiplication if $Y \otimes X \in \mathcal{O}$ (resp. $X \otimes Y \in \mathcal{O}$ ) for all $X \in \mathcal{O}$ and $Y \in \mathcal{C}$.

By a left (resp. right) ideal of a monoidal category $\mathcal{C}$, we mean a class $\mathcal{I} \subset \mathcal{C}$ which is closed under retraction and under left (resp. right) multiplication. By an ideal of a monoidal category $\mathcal{\ell}$, we mean a class $\mathcal{I} \subset \mathcal{C}$ which is both a left and right ideal.

Note that the closure under retraction implies that a left (resp. right) ideal $\mathcal{I}$ of a monoidal category $\mathcal{C}$ is replete, meaning that if $Y \in \mathcal{C}$ is isomorphic (in $\mathcal{C}$ ) to some $X \in \mathcal{I}$, then $Y \in \mathcal{I}$. In particular, if $\mathcal{C}$ is braided, then any left (resp. right) ideal is a (two-sided) ideal.

Lemma 1. Let $C$ be a pivotal category.

(a) A left (resp. right) ideal of ${ }^{C}$ is closed under biduality.

(b) An ideal of ${ }^{C}$ is closed under duality.

Proof. Part (a) follows from the facts that a left (resp. right) ideal is replete and that the bidual $X^{* *}$ of an object $X \in \mathcal{C}$ is isomorphic to $X$ (via the pivotal structure).

Let us prove (b). Let $\mathcal{I}$ be an ideal of $\mathcal{C}$. Given $X \in \mathcal{I}$, set $p=\mathrm{ev}_{X} \otimes \operatorname{id}_{X^{*}}$ and $q=\mathrm{id}_{X^{*}} \otimes \operatorname{coev}_{X}$. Then $p q=\mathrm{id}_{X^{*}}$. Thus $X^{*}$ is a retract of $X^{*} \otimes X \otimes X^{*} \in \mathcal{I}$ and so belongs to $\mathcal{I}$.

For a class of objects $\mathcal{I}$ in a pivotal category $\ell$, we set

$$
\mathcal{I}^{*}=\left\{Y \in \mathcal{C}: \exists X \in \mathcal{I}, Y \simeq X^{*}\right\} .
$$


Note that if $\mathcal{I}$ is a left or right ideal of $\mathcal{C}$, then

$$
\mathcal{I}^{*}=\left\{Y \in \mathcal{C}: Y^{*} \in \mathcal{I}\right\}
$$

(since ideals are replete and $Y \simeq Y^{* *}$ for any $Y \in \mathcal{C}$ ).

Lemma 2. Let $\mathcal{I}$ be a replete class of objects of a pivotal category $\mathcal{C}$.

(a) I is a left (resp. right) ideal if and only if $\mathcal{I}^{*}$ is a right (resp. left) ideal.

(b) $\mathcal{I}$ is an ideal if and only if $\mathcal{I}$ is a left (or right) ideal and $\mathcal{I}^{*}=\mathcal{I}$.

Proof. Part (a) follows from the fact that $(X \otimes Y)^{*} \simeq Y^{*} \otimes X^{*}$ and from the equivalence asserting that an object $U$ is a retract of an object $X$ if and only if $U^{*}$ is a retract of $X^{*}$. Let us prove (b). If $\mathcal{I}$ is a left (resp. right) ideal satisfying $\mathcal{I}=\mathcal{I}^{*}$ then, by (a), it is a right (resp. left) ideal, and so an ideal. Conversely if $\mathcal{I}$ is an ideal of $\mathcal{C}$, then $\mathcal{I}^{*}=\mathcal{I}$ by Lemma $1(\mathrm{~b})$.

2.2. Traces on ideals. Let $\mathcal{C}$ be a pivotal $\mathbb{k}$-category. A left trace on a left ideal $\mathcal{I}$ of $\mathcal{C}$ is a family $\mathrm{t}=\left\{\mathrm{t}_{X}: \operatorname{End}_{\mathcal{C}}(X) \rightarrow \mathbb{k}\right\}_{X \in \mathcal{I}}$ of $\mathbb{k}$-linear forms such that

$$
\mathrm{t}_{Y \otimes X}(f)=\mathrm{t}_{X}\left(\operatorname{tr}_{l}^{Y}(f)\right) \quad \text { and } \quad \mathrm{t}_{V}(g h)=\mathrm{t}_{U}(h g)
$$

for any $f \in \operatorname{Ende}_{e}(Y \otimes X), g \in \operatorname{Hom}_{e}(U, V)$, and $h \in \operatorname{Hom}_{e}(V, U)$, with $X, U, V \in$ $\mathcal{I}$ and $Y \in \boldsymbol{C}$.

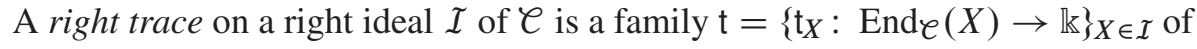
$\mathbb{k}$-linear forms such that

$$
\mathrm{t}_{X \otimes Z}(f)=\mathrm{t}_{X}\left(\operatorname{tr}_{r}^{Z}(f)\right) \text { and } \mathrm{t}_{V}(g h)=\mathrm{t}_{U}(h g)
$$

for any $f \in \operatorname{End}_{e}(X \otimes Z), g \in \operatorname{Hom}_{e}(U, V)$, and $h \in \operatorname{Hom}_{e}(V, U)$, with $X, U, V \in$ $\mathcal{I}$ and $Z \in \mathcal{C}$.

A trace on a ideal $\mathcal{I}$ of $\mathcal{C}$ is a family $\mathrm{t}=\left\{\mathrm{t}_{X}: \operatorname{End} \boldsymbol{C}(X) \rightarrow \mathbb{k}\right\}_{X \in \mathcal{I}}$ of $\mathbb{k}$-linear forms which is both a left and right trace on $\mathcal{I}$. Note that a trace $t$ on an ideal $\mathcal{I}$ satisfies

$$
\mathrm{t}_{Y \otimes X \otimes Z}(f)=\mathrm{t}_{X}\left(\operatorname{tr}_{l}^{Y} \operatorname{tr}_{r}^{Z}(f)\right)
$$

for any $f \in \operatorname{Ende}(Y \otimes X \otimes Z)$ with $X \in \mathcal{I}$ and $Y, Z \in \mathcal{C}$.

Lemma 3. The trace satisfies the following properties.

(a) If $\mathrm{t}$ is a left (resp. right) trace on a left (resp. right) ideal $\mathcal{I}$ of $\mathcal{C}$, then

$$
\mathrm{t}_{X^{* *}}\left(f^{* *}\right)=\mathrm{t}_{X}(f)
$$

for all $X \in \mathcal{I}$ and $f \in \operatorname{Ende}(X)$. 
(b) If $\mathrm{t}$ is a trace on an ideal I of $\mathcal{C}$, then

$$
\mathrm{t}_{X} *\left(f^{*}\right)=\mathrm{t}_{X}(f)
$$

for all $X \in \mathcal{I}$ and $f \in \operatorname{Ende}(X)$.

(c) If $\mathrm{t}$ is a left (resp. right) trace on a left (resp. right) ideal $\mathcal{I}$ of $\mathcal{C}$, then the family of $\mathbb{k}$-linear forms $\mathrm{t}^{\vee}=\left\{\mathrm{t}_{X}^{\vee}: \operatorname{End}_{\mathcal{C}}(X) \rightarrow \mathbb{k}\right\}_{X \in \mathcal{I}^{*}}$, defined by

$$
\mathrm{t}_{X}^{\vee}(f)=\mathrm{t}_{X} *\left(f^{*}\right)
$$

is a right (resp. left) trace on $\mathcal{I}^{*}$.

Proof. Let us prove (a). Denote by $\varphi$ the pivotal structure of $\varphi$ (see Section 1.1). Recall $X^{* *} \in \mathcal{I}$ (see Lemma 1) and $f^{* *}=\varphi_{X} f \varphi_{X}^{-1}$. Therefore,

$$
\mathrm{t}_{X^{* *}}\left(f^{* *}\right)=\mathrm{t}_{X^{* *}}\left(\varphi_{X} f \varphi_{X}^{-1}\right)=\mathrm{t}_{X}\left(f \varphi_{X}^{-1} \varphi_{X}\right)=\mathrm{t}_{X}(f) .
$$

Let us prove (b). Recall that $X^{*} \in \mathcal{I}$ (see Lemma 1). In particular, $X^{*} \otimes X \in \mathcal{I}$ and $X^{*} \otimes X \otimes X^{*} \in \mathcal{I}$. Set

$$
g=\left(\mathrm{ev}_{X} \otimes \mathrm{id}_{X^{*}}\right)\left(\mathrm{id}_{X} * \otimes f \otimes \mathrm{id}_{X^{*}}\right)
$$

and

$$
h=\mathrm{id}_{X} * \otimes \operatorname{coev}_{X}
$$

We have $g h=f^{*}$ and $\operatorname{tr}_{l}^{X^{*}}\left(\operatorname{tr}_{r}^{X^{*}}(h g)\right)=f$. Therefore,

$$
\begin{aligned}
\mathrm{t}_{X^{*}}\left(f^{*}\right) & =\mathrm{t}_{X^{*}}(g h) \\
& =\mathrm{t}_{X^{*} \otimes X \otimes X^{*}}(h g) \\
& =\mathrm{t}_{X^{*} \otimes X}\left(\operatorname{tr}_{r}^{X^{*}}(h g)\right) \\
& =\mathrm{t}_{X}\left(\operatorname{tr}_{l}^{X^{*}}\left(\operatorname{tr}_{r}^{X^{*}}(h g)\right)\right) \\
& =\mathrm{t}_{X}(f)
\end{aligned}
$$

by the properties of a (two-sided) trace.

Let us prove the left version of (c), from which the right version can be deduced by using $\ell^{\text {rev }}$. For $g \in \operatorname{Hom}_{e}(U, V)$ and $h \in \operatorname{Hom}_{e}(V, U)$, with $U, V \in \mathcal{I}^{*}$, we have

$$
\mathrm{t}_{V}^{\vee}(g h)=\mathrm{t}_{V^{*}}\left((g h)^{*}\right)=\mathrm{t}_{V^{*}}\left(h^{*} g^{*}\right)=\mathrm{t}_{U^{*}}\left(g^{*} h^{*}\right)=\mathrm{t}_{U^{*}}\left((h g)^{*}\right)=\mathrm{t}_{U}^{\vee}(h g) .
$$


Now let $f \in \operatorname{End}_{e}(Y \otimes Z)$ with $X \in \mathcal{I}^{*}$ and $Z \in \mathcal{C}$. Let $\varphi:(X \otimes Z)^{*} \rightarrow Z^{*} \otimes X^{*}$ be the canonical isomorphism. Then $\operatorname{tr}_{l}^{Z^{*}}\left(\varphi f^{*} \varphi^{-1}\right)=\left(\operatorname{tr}_{r}^{Z}(f)\right)^{*}$ and so

$$
\begin{aligned}
\mathrm{t}_{X \otimes Z}^{\vee}(f) & =\mathrm{t}_{(X \otimes Z)^{*}}\left(f^{*}\right) \\
& =\mathrm{t}_{Z^{*} \otimes X^{*}}\left(\varphi f^{*} \varphi^{-1}\right) \\
& =\mathrm{t}_{X^{*}}\left(\operatorname{tr}_{r}^{Z^{*}}\left(\varphi f^{*} \varphi^{-1}\right)\right)=\mathrm{t}_{X^{*}}\left(\left(\operatorname{tr}_{r}^{Z}(f)\right)^{*}\right) \\
& =\mathrm{t}_{X}^{\vee}\left(\operatorname{tr}_{r}^{Z}(f)\right)
\end{aligned}
$$

since $t$ is a left trace and $X^{*} \in \mathcal{I}$. Hence, $\mathrm{t}^{\vee}$ is a right trace.

Lemma 4. Let $\mathcal{C}$ be a pivotal $\mathbb{k}$-category. Denote by $\varphi$ the pivotal structure of $\mathcal{C}$ (see Section 1.1).

(a) If $\mathrm{t}$ is a left trace on a left ideal I of $\mathcal{C}$ then

$$
\mathrm{t}_{X}\left(\varphi_{X}^{-1}\left(\operatorname{tr}_{l}^{X^{\prime}}(f)\right)^{*} \varphi_{X}\right)=\mathrm{t}_{X^{\prime}}\left(\operatorname{tr}_{r}^{X^{*}}(f)\right)
$$

for all $X, X^{\prime} \in \mathcal{I}$ and $f \in \operatorname{End}_{e}\left(X^{\prime} \otimes X^{*}\right)$.

(b) If $\mathrm{t}$ is a right trace on a right ideal $\mathcal{I}$ of $\mathcal{C}$ then

$$
\mathrm{t}_{X}\left(\varphi_{X}^{-1}\left(\operatorname{tr}_{r}^{X^{\prime}}(g)\right)^{*} \varphi_{X}\right)=\mathrm{t}_{X^{\prime}}\left(\operatorname{tr}_{l}^{X^{*}}(g)\right)
$$

for all $X, X^{\prime} \in \mathcal{I}$ and $g \in \operatorname{End} e\left(X^{*} \otimes X^{\prime}\right)$.

(c) If $\mathrm{t}$ is a trace on an ideal $\mathcal{I}$ of $\mathcal{C}$ then

$$
\mathrm{t}_{X}\left(\varphi_{X}^{-1}\left(\operatorname{tr}_{l}^{X^{\prime} \otimes Y}(f)\right)^{*} \varphi_{X}\right)=\mathrm{t}_{X^{\prime}}\left(\operatorname{tr}_{r}^{Y \otimes X^{*}}(f)\right),
$$

and

$$
\mathrm{t}_{X}\left(\varphi_{X}^{-1}\left(\operatorname{tr}_{r}^{Y \otimes X^{\prime}}(g)\right)^{*} \varphi_{X}\right)=\mathrm{t}_{X^{\prime}}\left(\operatorname{tr}_{l}^{X^{*} \otimes Y}(g)\right),
$$

for all $X, X^{\prime} \in \mathcal{I}$, and $Y \in \mathcal{C}$ and for all $f \in \operatorname{End}_{\ell}\left(X^{\prime} \otimes Y \otimes X^{*}\right)$, and $g \in \operatorname{End}_{e}\left(X^{*} \otimes Y \otimes X^{\prime}\right)$.

Proof. Let us prove (a). Let $f \in \operatorname{End} C\left(X^{\prime} \otimes X^{*}\right)$ where $X, X^{\prime} \in \mathcal{I}$. Set

$$
\begin{aligned}
& \alpha=\left(\operatorname{id}_{X^{\prime *}} \otimes \operatorname{id}_{X^{\prime}} \otimes \widetilde{\mathrm{ev}}_{X^{*}}\right)\left(\mathrm{id}_{X^{\prime *}} \otimes f \otimes \operatorname{id}_{X^{* *}}\right)\left(\widetilde{\operatorname{cov}}_{X^{\prime}} \otimes \operatorname{id}_{X^{*}} \otimes \varphi_{X}\right)
\end{aligned}
$$

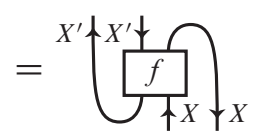

and

$$
\beta=\left(\operatorname{id}_{X^{*}} \otimes \varphi_{X}^{-1}\right) \operatorname{coev}_{X^{*}} \operatorname{ev}_{X^{\prime}}=\bigcup_{\bigcap_{X^{\prime}}} .
$$


Since $X^{*} \otimes X \in \mathcal{I}, X^{*} \otimes X^{\prime} \in \mathcal{I}$, and $\mathrm{t}$ is a left trace, we have

$$
\mathrm{t}_{X}\left(\operatorname{tr}_{l}^{X^{*}}(\beta \alpha)\right)=\mathrm{t}_{X^{*} \otimes X}(\beta \alpha)=\mathrm{t}_{X^{\prime *} \otimes X^{\prime}}(\alpha \beta)=\mathrm{t}_{X^{\prime}}\left(\operatorname{tr}_{l}^{X^{\prime *}}(\alpha \beta)\right) .
$$

Now

$$
\operatorname{tr}_{l}^{X^{*}}(\beta \alpha)={\stackrel{x}{X^{\prime}} \curvearrowleft \overbrace{X}}^{\prime f}=\varphi_{X}^{-1}\left(\operatorname{tr}_{l}^{X^{\prime}}(f)\right)^{*} \varphi_{X}
$$

and

$$
\operatorname{tr}_{l}^{X^{\prime *}}(\alpha \beta)=\underset{X^{\prime}}{f} f^{X}=\operatorname{tr}_{r}^{X^{*}}(f) .
$$

Therefore, (4) is satisfied. We deduce (b) from (a) by using $e^{\text {rev }}$.

Let us prove (c). Let $f \in \operatorname{End}_{\mathcal{C}}\left(X^{\prime} \otimes Y \otimes X^{*}\right)$, where $X, X^{\prime} \in \mathcal{I}$ and $Y \in \mathcal{C}$. Set

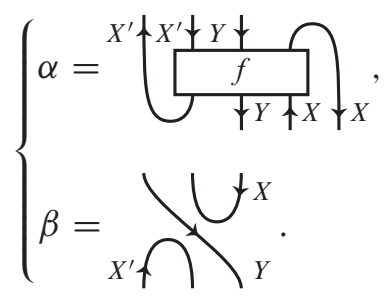

Since $Y \otimes X^{*} \otimes X \in \mathcal{I}, X^{\prime *} \otimes X^{\prime} \otimes Y \in \mathcal{I}$, and $\mathrm{t}$ is a trace, we have

$$
\mathrm{t}_{X}\left(\operatorname{tr}_{l}^{Y \otimes X^{*}}(\beta \alpha)\right)=\mathrm{t}_{Y \otimes X^{*} \otimes X}(\beta \alpha)=\mathrm{t}_{X^{\prime *} \otimes X^{\prime} \otimes Y}(\alpha \beta)=\mathrm{t}_{X^{\prime}}\left(\operatorname{tr}_{l}^{X^{\prime *}} \operatorname{tr}_{r}^{Y}(\alpha \beta)\right) .
$$

Now

$$
\operatorname{tr}_{l}^{Y \otimes X^{*}}(\beta \alpha)=\underbrace{X}_{Y}
$$

and

$$
\operatorname{tr}_{l}^{X^{\prime *}} \operatorname{tr}_{r}^{Y}(\alpha \beta)=\overbrace{X^{\prime}}^{X^{\prime}+}\}^{Y} .
$$


Therefore, t satisfies (6). Likewise, given $g \in$ Ende $\left(X^{*} \otimes Y \otimes X^{\prime}\right)$, by using

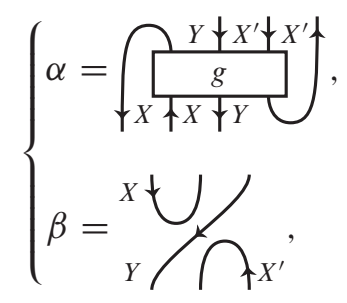

we obtain that $t$ satisfies (7).

2.3. Modified dimensions. If $t$ is a left (resp. right) trace on a left (resp. right) ideal $\mathcal{I}$ in a pivotal $\mathbb{k}$-category $\mathcal{C}$, the left (resp. right) modified dimension (associated with $\mathrm{t}$ ) is the function defined on the objects $V \in \mathcal{I}$ by

$$
\mathrm{d}_{l}(V)=\mathrm{t}_{V}\left(\mathrm{id}_{V}\right) \quad\left(\text { resp. } \mathrm{d}_{r}(V)=\mathrm{t}_{V}\left(\operatorname{id}_{V}\right)\right) .
$$

Note that an immediate consequence of the definition of a left trace (resp. right trace) is that isomorphic objects have equal modified dimensions.

Let $\mathrm{t}$ be a trace on an ideal $\mathcal{I}$ in $\mathcal{C}$, the modified dimension (associated with $\mathrm{t}$ ) is the function defined on the objects $V \in \mathcal{I}$ by

$$
\mathrm{d}(V)=\mathrm{t}_{V}\left(\operatorname{id}_{V}\right) .
$$

Note that if $V \in \mathcal{I}$, then $V^{*} \in \mathcal{I}$ and $\mathrm{d}(V)=\mathrm{d}\left(V^{*}\right)$ by Lemmata 1 and 3 .

\section{Invariants of closed graphs}

3.1. Colored graphs. Let $\mathcal{C}$ be a pivotal category. By a $\mathcal{C}$-colored ribbon graph in an oriented surface $\Sigma$, we mean a graph embedded in $\Sigma$ whose edges are oriented and colored by objects of $\mathcal{C}$ and whose vertices lying in Int $\Sigma=\Sigma-\partial \Sigma$ are thickened to coupons colored by morphisms of $\mathcal{C}$ (as in Penrose graphical calculus, see Section 1.3). The edges of a $\mathcal{C}$-colored graph do not meet each other and may meet the coupons only at the bottom and top sides. The intersection of a $\mathscr{C}$-colored ribbon graph in $\Sigma$ with $\partial \Sigma$ is required to be empty or to consist only of vertices of valency 1 .

A $\mathcal{C}$-colored ribbon graph in $\mathbb{R}^{2}$ (with counterclockwise orientation) is called planar. A $\mathcal{C}$-colored ribbon graph in $S^{2}=\mathbb{R}^{2} \cup\{\infty\}$ is called spherical.

The $\mathcal{C}$-colored ribbon graphs in $\mathbb{R} \times[0,1]$ (with counterclockwise orientation) form a category $\mathscr{G}_{e}$ as follows: objects of $\mathscr{G}_{\mathcal{E}}$ are finite sequences of pairs $(X, \varepsilon)$, where $X \in \mathcal{C}$ and $\varepsilon= \pm$. Morphisms of $\mathscr{C}_{\mathcal{C}}$ are isotopy classes of $\mathcal{C}$-colored ribbon graphs in $\mathbb{R} \times[0,1]$. Composition, identities, tensor multiplication, left and right duality in $\mathscr{C}_{e}$ are defined in the standard way. In particular,

$$
\left(\left(V_{1}, \varepsilon_{1}\right), \ldots,\left(V_{n}, \varepsilon_{n}\right)\right)^{*}=\left(\left(V_{n},-\varepsilon_{n}\right), \ldots,\left(V_{1},-\varepsilon_{1}\right)\right)
$$


and the dual $T^{*}$ of a $\mathcal{C}$-colored ribbon graph $T$ in $\mathbb{R} \times[0,1]$ is obtained by rotating $T$ by $\pi$. For example, if

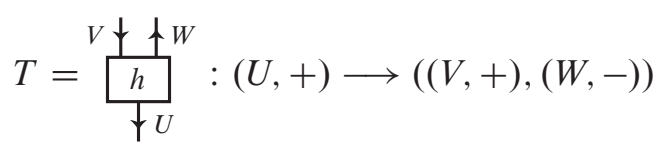

where $h \in \mathrm{Hom}_{\mathcal{C}}(U, V \otimes W)$, then

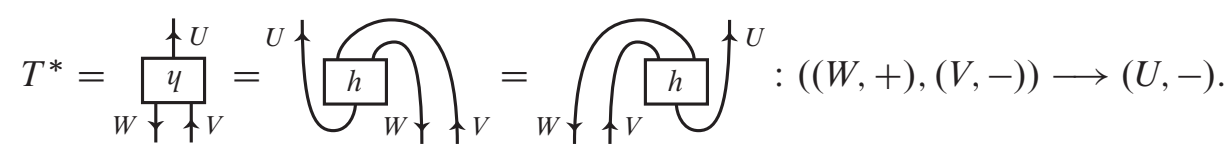

This makes $\mathscr{C}_{e}$ into a pivotal category. By Penrose graphical calculus, one obtains a (strict) monoidal functor

$$
F: \varepsilon_{e} \rightarrow e \text {. }
$$

In particular, $F$ sends $(X, \varepsilon) \in \mathrm{Ob}\left(\mathscr{C}_{\mathscr{C}}\right)$ to

$$
X^{\varepsilon}= \begin{cases}X & \text { if } \varepsilon=+, \\ X^{*} & \text { if } \varepsilon=-\end{cases}
$$

By a $\mathcal{C}$-colored 1-1-ribbon graph, we mean an endomorphism $T$ in $\mathscr{E}$ of a pair $(X, \varepsilon)$, where $X \in \mathcal{C}$ and $\varepsilon= \pm$. The pair $(X, \varepsilon)$ is called the section of $T$. The left

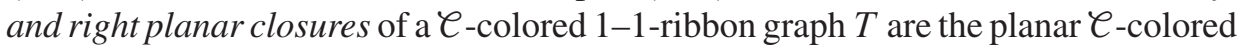
ribbon graphs (embedded in the interior of $\mathbb{R} \times[0,1]$ ) defined by

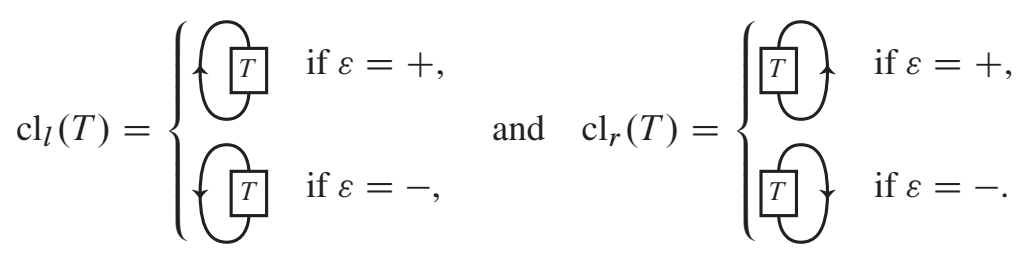

Note that $F\left(\mathrm{cl}_{l}(T)\right)=\operatorname{tr}_{l}(F(T))$ and $F\left(\mathrm{cl}_{r}(T)\right)=\operatorname{tr}_{r}(F(T))$.

By a left (resp. right) cutting presentation of a planar $\mathcal{C}$-colored graph $\Gamma$, we mean a $\mathcal{C}$-colored 1-1-ribbon graph $T$ such that $\Gamma=\operatorname{cl}_{l}(T)\left(\operatorname{resp} . \Gamma=\operatorname{cl}_{r}(T)\right)$.

Considered as spherical $\mathcal{C}$-colored ribbon graphs, the left and right planar closures of a $\mathcal{C}$-colored 1-1-ribbon graph $T$ are isotopic, and are called the spherical closure of $T$ and denoted $\operatorname{cl}(T)$. By a cutting presentation of a spherical $\mathcal{C}$-colored graph $\Gamma$, we mean a $\mathcal{C}$-colored 1-1-ribbon graph $T$ such that $\Gamma=\operatorname{cl}(T)$.

3.2. Invariants of admissible graphs. Let $\mathcal{C}$ be a pivotal $\mathbb{k}$-category and $\mathcal{I}$ be a left (resp. right) ideal of $\mathcal{C}$. A $\mathcal{C}$-colored 1-1-ribbon graph is left (resp. right) $\mathcal{I}$-admissible if its section $(V, \varepsilon)$ has the property that $V^{\varepsilon} \in \mathcal{I}$. By a left (resp. right) $\mathcal{I}$-admissible planar graph we mean a planar $\mathcal{C}$-colored ribbon graphs which admits a left (resp. right) $\mathcal{I}$-admissible left (resp. right) cutting presentation. 
If t is a left (resp. right) trace on $\mathcal{I}$ and $\Gamma$ is a left (resp. right) $\mathcal{I}$-admissible planar graph, we set

$$
F_{\mathrm{t}}^{l}(\Gamma)=\mathrm{t}_{V^{\varepsilon}}(F(T)) \quad\left(\text { resp. } F_{\mathrm{t}}^{r}(\Gamma)=\mathrm{t}_{V^{\varepsilon}}(F(T))\right),
$$

where $T$ is a left (resp. right) $\mathcal{I}$-admissible left (resp. right) cutting presentation of $\Gamma$ with section $(V, \varepsilon)$.

When $\mathcal{I}$ is an ideal, by a $\mathcal{I}$-admissible spherical graph, we mean spherical $\zeta$-colored ribbon graph such that at least one of its edges is colored by an element of $\mathcal{I}$. Note that such a graph admits a cutting presentation with section $(V,+)$ with $V \in \mathcal{I}$.

Theorem 5. Let $\mathrm{t}$ be a left (resp. right) trace on a left (resp. right) ideal $\mathcal{I}$ of $\mathcal{C}$. Then $F_{\mathrm{t}}^{l}$ (resp. $\left.F_{\mathrm{t}}^{r}\right)$ is an isotopy invariant of left (resp. right) $\mathcal{I}$-admissible graphs. Moreover, if $\mathcal{I}$ is an ideal and $\mathrm{t}$ is a trace on $\mathcal{I}$, then $F_{\mathrm{t}}^{l}=F_{\mathrm{t}}^{r}$ and this invariant extends to an isotopy invariant of $\mathcal{I}$-admissible spherical graphs, denoted $F_{\mathrm{t}}$.

When $\mathcal{I}=\mathcal{C}$ and $\mathrm{t}$ is the usual left (resp. right) trace of endomorphisms of $\mathcal{C}$, then $F_{\mathrm{t}}^{l}$ (resp. $F_{\mathrm{t}}^{r}$ ) is nothing but the usual invariant obtained by Penrose calculus.

Before proving Theorem 5, we introduce some notation. Remark first that if $T:\left(\left(V_{1}, \varepsilon_{1}\right), \ldots,\left(V_{m}, \varepsilon_{m}\right)\right) \rightarrow\left(\left(V_{1}^{\prime}, \varepsilon_{1}^{\prime}\right), \ldots,\left(V_{n}^{\prime}, \varepsilon_{n}^{\prime}\right)\right)$ is a morphism in $\mathscr{E}_{e}$, then

$$
F\left(T^{*}\right)=\left(\psi_{\left(V_{m}, \varepsilon_{m}\right)}^{-1} \otimes \cdots \otimes \psi_{\left(V_{1}, \varepsilon_{1}\right)}^{-1}\right) \circ F(T)^{*} \circ\left(\psi_{\left(V_{n}^{\prime}, \varepsilon_{n}^{\prime}\right)} \otimes \cdots \otimes \psi_{\left(V_{1}^{\prime}, \varepsilon_{1}^{\prime}\right)}\right)
$$

where $\psi_{(V, \varepsilon)}: V^{-\varepsilon} \rightarrow\left(V^{\varepsilon}\right)^{*}$ is the isomorphism given by

and

$$
\psi_{(V,-1)}=\varphi_{V}: V \longrightarrow V^{* *}
$$

$$
\psi_{(V, 1)}=\mathrm{id}_{V^{*}}: V^{*} \longrightarrow V^{*}
$$

Let $T$ be an endomorphism of $\left(\left(V_{1}, \varepsilon_{1}\right), \ldots,\left(V_{m}, \varepsilon_{m}\right)\right)$ in $\mathscr{E}$. By taking the left closure of the $k$ left most strands of $T$, we define the partial left closure of $T$ denoted by

$$
\mathrm{cl}_{l}^{\left(\left(V_{1}, \varepsilon_{1}\right), \ldots,\left(V_{k}, \varepsilon_{k}\right)\right)}(T) \in \operatorname{End}_{\mathscr{e}_{e}}\left(\left(V_{k+1}, \varepsilon_{k+1}\right), \ldots,\left(V_{n}, \varepsilon_{n}\right)\right) .
$$

Similarly, by taking the right closure of the $k$ right most strands of $T$, we define the partial right closure of $T$ denoted by

$$
\mathrm{cl}_{r}^{\left(\left(V_{n-k+1}, \varepsilon_{n-k+1}\right), \ldots,\left(V_{n}, \varepsilon_{n}\right)\right)}(T) \in \operatorname{End} \mathscr{E}_{e}\left(\left(V_{1}, \varepsilon_{1}\right), \ldots,\left(V_{n-k}, \varepsilon_{n-k}\right)\right) .
$$

Let $\Gamma$ be a spherical $C$-colored ribbon graph in $S^{2}=\mathbb{R}^{2} \cup\{\infty\}$. By a cutting path of $\Gamma$ we mean an embedded oriented path $\gamma:[0,1] \rightarrow S^{2}$ such that $\gamma(0), \gamma(1) \notin \Gamma$ and whose image does not meet the coupons of $\Gamma$ and meets any edge of $\Gamma$ transversally. Let $\gamma$ be a cutting path of $\Gamma$. Consider a tubular neighborhood $\Omega \simeq \gamma([0,1]) \times]-1,1[$ of the image $\gamma([0,1])$ of $\gamma$. We identify $S^{2} \backslash \Omega \simeq \mathbb{R} \times[0,1]$ in such a way that the 
boundary components $\gamma(] 0,1[) \times\{-1\}$ and $\gamma(] 0,1[) \times\{1\}$ of $S^{2} \backslash \Omega$ are sent to the top boundary $\mathbb{R} \times\{1\}$ and the bottom boundary $\mathbb{R} \times\{0\}$ of $\mathbb{R} \times[0,1]$, respectively. Then we denote by $\operatorname{cut}_{\gamma}(\Gamma)$ the endomorphism of $\mathscr{E}$ e equal to $\Gamma \backslash \Omega \subset S^{2} \backslash \Omega \simeq \mathbb{R} \times[0,1]$.

If $\Gamma$ is a planar $\ell$-colored ribbon graph, considered as a spherical $\ell$-colored graph in $S^{2}=\mathbb{R}^{2} \cup\{\infty\}$, and $\gamma$ is a cutting path for $\Gamma$ such that the points $\gamma(1)$ (resp. $\gamma(0)$ ) and $\infty$ are in the same component of $S^{2} \backslash \Gamma$, then $\operatorname{cl}_{l}\left(\operatorname{cut}_{\gamma}(\Gamma)\right)\left(\operatorname{resp} \operatorname{cl}_{r}\left(\operatorname{cut}_{\gamma}(\Gamma)\right)\right)$ and $\Gamma$ are isotopic.

Proof of Theorem 5. Let us prove the first statement of the theorem. Let $t$ be a left (resp. right) trace on a left (resp. right) ideal $\mathcal{I}$. Let $\Gamma$ be an $\mathcal{I}$-admissible planar $\mathcal{C}$-colored ribbon graph and $T_{0}, T_{1}$ be two left cutting presentations of $\Gamma$ with sections $\left(V_{0}, \varepsilon_{0}\right)$ and $\left(V_{1}, \varepsilon_{1}\right)$, respectively. We have to show that $\mathrm{t}_{V^{\varepsilon_{0}}}\left(F\left(T_{0}\right)\right)=$ $\mathrm{t}_{V_{1}^{\varepsilon_{1}}}\left(F\left(T_{1}\right)\right)$. The two edges $e_{0}$ and $e_{1}$ of $\Gamma$ that are cut to form $T_{0}$ and $T_{1}$, respectively, are in the boundary of the unbounded component $C$ of $\mathbb{R}^{2} \backslash \Gamma$. Let $\gamma_{0}$ and $\gamma_{1}$ be two disjoint cutting paths located in a neighborhoods of $e_{0}$ and $e_{1}$, respectively, such that $T_{i}=\operatorname{cut}_{\gamma_{i}}(\Gamma)$ and $\gamma_{i}(1) \in C$ for $i=0,1$. Choose an embedded path $\gamma_{2}:[0,1] \rightarrow C \backslash\left(\gamma_{0}(] 0,1[) \cup \gamma_{1}(] 0,1[)\right)$ such that $\gamma_{2}(0)=\gamma_{0}(1)$ and $\gamma_{2}(1)=\gamma_{1}(1)$. Define $\gamma$ as the concatenation of paths $\gamma=\bar{\gamma}_{1} \gamma_{2} \gamma_{0}$ where $\bar{\gamma}_{1}(t)=\gamma_{1}(1-t)$. Set

$$
T=\operatorname{cut}_{\gamma}(\Gamma) \in \operatorname{End}_{\varepsilon_{e}}\left(\left(V_{0}, \varepsilon_{0}\right),\left(V_{1},-\varepsilon_{1}\right)\right) .
$$

When $\varepsilon_{0}=\varepsilon_{1}=1$, the construction of $T$ can be schematically depicted as

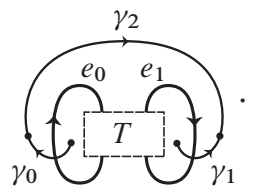

By construction, we have $T_{0}=\mathrm{cl}_{r}^{\left(V_{1},-\varepsilon_{1}\right)}(T)$ and $T_{1}=\mathrm{cl}_{r}^{\left(V_{0},-\varepsilon_{0}\right)}\left(T^{*}\right)$. Then, setting

$$
f=\left(\operatorname{id}_{V_{0}^{\varepsilon_{0}}} \otimes \psi_{\left(V_{1}, \varepsilon_{1}\right)}\right) F(T)\left(\operatorname{id}_{V_{0}^{\varepsilon_{0}}} \otimes \psi_{\left(V_{1}, \varepsilon_{1}\right)}^{-1}\right) \in \operatorname{End}_{e}\left(V_{0}^{\varepsilon_{0}} \otimes\left(V_{1}^{\varepsilon_{1}}\right)^{*}\right),
$$

we have

$$
\begin{aligned}
\mathrm{t}_{V_{0}^{\varepsilon_{0}}}\left(F\left(T_{0}\right)\right) & =\mathrm{t}_{V_{0}^{\varepsilon_{0}}}\left(F\left(\mathrm{cl}_{r}^{\left(V_{1},-\varepsilon_{1}\right)}(T)\right)\right) \\
& =\mathrm{t}_{V_{0}^{\varepsilon_{0}}}\left(\operatorname{tr}_{r}^{V_{1}^{-\varepsilon_{1}}}(F(T))\right) \\
& =\mathrm{t}_{V_{0}^{\varepsilon_{0}}}\left(\operatorname{tr}_{r}^{V_{1}^{-\varepsilon_{1}}}\left(\left(\operatorname{id}_{V_{0}^{\varepsilon_{0}}} \otimes \psi_{\left(V_{1}, \varepsilon_{1}\right)}^{-1}\right) f\left(\operatorname{id}_{V_{0}^{\varepsilon_{0}}} \otimes \psi_{\left(V_{1}, \varepsilon_{1}\right)}\right)\right)\right) \\
& =\mathrm{t}_{V_{0}^{\varepsilon_{0}}}\left(\operatorname{tr}_{r}{ }^{\left.\varepsilon_{1}^{\varepsilon_{1}}\right)^{*}}(f)\right)
\end{aligned}
$$

and

$$
\mathrm{t}_{V_{1}^{\varepsilon} \varepsilon_{1}}\left(F\left(T_{1}\right)\right)=\mathrm{t}_{V_{1}^{\varepsilon} \varepsilon_{1}}\left(F\left(\mathrm{cl}_{r}^{\left(V_{0},-\varepsilon_{0}\right)}\left(T^{*}\right)\right)\right)=\mathrm{t}_{V_{1}^{\varepsilon_{1}}}\left(\operatorname{tr}_{r}^{V_{0}^{-\varepsilon_{0}}}\left(F\left(T^{*}\right)\right)\right) .
$$


Now, we have

$$
\begin{aligned}
F\left(T^{*}\right) & =\left(\psi_{\left(V_{1},-\varepsilon_{1}\right)}^{-1} \otimes \psi_{\left(V_{0}, \varepsilon_{0}\right)}^{-1}\right) F(T)^{*}\left(\psi_{\left(V_{1},-\varepsilon_{1}\right)} \otimes \psi_{\left(V_{0}, \varepsilon_{0}\right)}\right) \\
& =\left(\varphi_{V_{1}^{\varepsilon}}^{-1} \otimes \psi_{\left(V_{0}, \varepsilon_{0}\right)}^{-1}\right) f^{*}\left(\varphi_{V_{1}^{\varepsilon_{1}}} \otimes \psi_{\left(V_{0}, \varepsilon_{0}\right)}\right)
\end{aligned}
$$

by using (8) and the fact that $\psi_{(V, \varepsilon)}^{*}=\psi_{\left(V^{*}, \varepsilon\right)}^{-1}$. Therefore

$$
\begin{aligned}
\operatorname{tr}_{r}^{V_{0}^{-\varepsilon_{0}}}\left(F\left(T^{*}\right)\right) & =\varphi_{V_{1}^{\varepsilon_{1}}}^{-1} \operatorname{tr}_{r}^{V_{0}^{-\varepsilon_{0}}}\left(\left(\mathrm{id} \otimes \psi_{V_{0}, \varepsilon_{0}}^{-1}\right) f^{*}\left(\mathrm{id} \otimes \psi_{V_{0}, \varepsilon_{0}}\right)\right) \varphi_{V_{1}^{\varepsilon_{1}}} \\
& =\varphi_{V_{1}^{\varepsilon_{1}}}^{-1} \operatorname{tr}_{r}^{\left(V_{0}^{\varepsilon_{0}}\right)^{*}}\left(f^{*}\right) \varphi_{V_{1}^{\varepsilon_{1}}} \\
& =\varphi_{V_{1}^{\varepsilon_{1}}}^{-1}\left(\operatorname{tr}_{l}^{V_{0}^{\varepsilon_{0}}}(f)\right)^{*} \varphi_{V_{1}^{\varepsilon_{1}}}
\end{aligned}
$$

and so

$$
\mathrm{t}_{V_{1}^{\varepsilon_{1}}}\left(F\left(T_{1}\right)\right)=\mathrm{t}_{V_{1}^{\varepsilon_{1}}}\left(\operatorname{tr}_{r}^{V_{0}^{-\varepsilon_{0}}}\left(F\left(T^{*}\right)\right)\right)=\mathrm{t}_{V_{1}^{\varepsilon_{1}}}\left(\varphi_{V_{1}^{\varepsilon_{1}}}^{-1}\left(\operatorname{tr}_{l}^{V_{0}^{\varepsilon_{0}}}(f)\right)^{*} \varphi_{V_{1}^{\varepsilon_{1}}}\right)
$$

Finally, by Lemma 4(a), we have

$$
\mathrm{t}_{V_{1}^{\varepsilon_{1}}}\left(F\left(T_{1}\right)\right)=\mathrm{t}_{V_{1}^{\varepsilon_{1}}}\left(\varphi_{V_{1}^{\varepsilon_{1}}}^{-1}\left(\operatorname{tr}_{l}^{V_{0}^{\varepsilon_{0}}}(f)\right)^{*} \varphi_{V_{1}^{\varepsilon_{1}}}\right)=\mathrm{t}_{V_{0}^{\varepsilon_{0}}}\left(\operatorname{tr}_{r}^{\left(V_{1}^{\varepsilon_{1}}\right)^{*}}(f)\right)=\mathrm{t}_{V_{0}^{\varepsilon_{0}}}\left(F\left(T_{0}\right)\right) .
$$

Thus, $F_{\mathrm{t}}$ is an isotopy invariant of left $\mathcal{I}$-admissible graphs. Then using $\mathcal{C}^{\text {rev }}$ it follows that $F_{\mathrm{t}}^{r}$ is an isotopy invariant of right $\mathcal{I}$-admissible graphs when $\mathrm{t}$ is a left trace. This concludes the proof of the first statement in Theorem 5.

We now prove the second statement of the theorem. Let $t$ be a trace on an ideal $\mathcal{I}$. Let $\Gamma$ be an $\mathcal{I}$-admissible spherical $\mathcal{C}$-colored ribbon graph and $T_{0}, T_{1}$ be two cutting presentations of $\Gamma$ with sections $(V, \varepsilon)$ and $\left(V^{\prime}, \varepsilon^{\prime}\right)$, respectively. We have to show that $\mathrm{t}_{V^{\prime} \varepsilon^{\prime}}\left(F\left(T_{1}\right)\right)=\mathrm{t}_{V^{\varepsilon}}\left(F\left(T_{0}\right)\right)$. Let $e_{0}$ and $e_{1}$ be the edges of $\Gamma$ that are cut to form $T_{0}$ and $T_{1}$, respectively. Let $\gamma_{0}$ and $\gamma_{1}$ be two disjoint cutting paths located in a neighborhoods of $e_{0}$ and $e_{1}$, respectively, such that $T_{i}=\operatorname{cut}_{\gamma_{i}}(\Gamma)$ for $i=0,1$. Choose an embedded path $\gamma_{2}:[0,1] \rightarrow S^{2} \backslash\left(\gamma_{0}\left(\left[0,1[) \cup \gamma_{1}\left([0,1[))\right.\right.\right.\right.$ such that $\gamma_{2}(0)=$ $\gamma_{0}(1), \gamma_{2}(1)=\gamma_{1}(1)$, and whose image does not meet the coupons of $\Gamma$ and meets any edge of $\Gamma$ transversally. Define $\gamma$ as the concatenation of paths $\gamma=\bar{\gamma}_{1} \gamma_{2} \gamma_{0}$, where $\bar{\gamma}_{1}(t)=\gamma_{1}(1-t)$. Set

$$
T=\operatorname{cut}_{\gamma}(\Gamma) \in \operatorname{End}_{\mathscr{E}_{e}}\left(\left(V_{0}, \varepsilon_{0}\right),\left(V_{1}, \varepsilon_{1}\right), \ldots,\left(V_{n}, \varepsilon_{n}\right)\right),
$$

where $\left(V_{0}, \varepsilon_{0}\right)=(V, \varepsilon),\left(V_{n}, \varepsilon_{n}\right)=\left(V^{\prime}, \varepsilon^{\prime}\right)$ and $V_{1}, \ldots, V_{n}$ are the colors of the edges met by $\gamma_{2}$. By construction,

$$
T_{0}=\mathrm{cl}_{r}^{\left(\left(V_{1}, \varepsilon_{1}\right), \ldots,\left(V_{n}, \varepsilon_{n}\right)\right)}(T)
$$


and

$$
T_{1}=\mathrm{cl}_{r}^{\left(\left(V_{n-1},-\varepsilon_{n-1}\right), \ldots,\left(V_{0},-\varepsilon_{0}\right)\right)}\left(T^{*}\right) .
$$

Let $Y=V_{1}^{\varepsilon_{1}} \otimes \cdots \otimes V_{n-1}^{\varepsilon_{n-1}}$ and set

$$
f=\left(\operatorname{id}_{V_{0}^{\varepsilon_{0}} \otimes Y} \otimes \psi_{V_{n}, \varepsilon_{n}}\right) F(T)\left(\operatorname{id}_{V_{0}^{\varepsilon_{0}} \otimes Y} \otimes \psi_{V_{n}, \varepsilon_{n}}^{-1}\right) \in \operatorname{End}_{e}\left(V_{0}^{\varepsilon_{0}} \otimes Y \otimes\left(V_{n}^{\varepsilon_{n}}\right)^{*}\right) .
$$

As in the first part of the theorem, one can show that

$$
\mathrm{t}_{V_{0}^{\varepsilon_{0}}}\left(F\left(T_{0}\right)\right)=\mathrm{t}_{V_{0}^{\varepsilon_{0}}}\left(\operatorname{tr}_{r}^{Y \otimes\left(V_{n}^{\varepsilon n}\right)^{*}}(f)\right)
$$

and

$$
\mathrm{t}_{V_{n}^{\varepsilon n}}\left(F\left(T_{1}\right)\right)=\mathrm{t}_{V_{n}^{\varepsilon n}}\left(\varphi_{V_{n}^{\varepsilon n}}^{-1}\left(\operatorname{tr}_{l}^{V_{0}^{\varepsilon_{0}} \otimes Y}(f)\right)^{*} \varphi_{V_{n}^{\varepsilon n}}\right)
$$

Thus, the second statement of theorem follows from these formulas and (6).

3.3. Invariants of spherical graphs from one-sided traces. Let $\varphi$ be a pivotal $\mathbb{k}$-category. By a A-colored graph, where $A$ is a class of object of $\mathcal{C}$, we mean a $\zeta$-colored graph whose edges are colored by elements of $A$.

Let $t$ be a left (resp. right) trace on a left (resp. right) ideal $\mathcal{I}$. Set

$$
\mathrm{A}=\left\{V \in \mathcal{I} \cap \mathcal{I}^{*}: \mathrm{t}_{V}=\mathrm{t}_{V}^{\vee}\right\}
$$

where $t^{\vee}$ is defined in Lemma 3(c). For any spherical A-colored ribbon graph $\Gamma$, set

$$
F_{\mathrm{t}}^{a}(\Gamma)=\mathrm{t}_{V^{\varepsilon}}(F(T))
$$

where $T$ is any cutting presentation of $\Gamma$ with section $(V, \varepsilon)$.

Theorem 6. $F_{\mathrm{t}}^{a}$ is an isotopy invariant of spherical A-colored ribbon graphs.

Note that if $\mathrm{t}$ is a trace on an ideal $\mathcal{I}$, then $A=\mathcal{I}$ (by Lemmata 1 and 3), and the invariants $F_{\mathrm{t}}$ and $F_{\mathrm{t}}^{a}$ coincide on spherical $\mathcal{I}$-colored ribbon graphs.

Proof. We prove Theorem 6 in the case when t is a left trace. Then the case when $t$ is a right trace can be deduced using $e^{\text {rev }}$. Let $\Gamma$ be a spherical A-colored ribbon graph and let $T$ be a cutting presentation of $\Gamma$. Then $\Gamma^{\prime}=\operatorname{cl}_{l}(T)$ is a planar A-colored ribbon graph whose isotopy class in $S^{2}=\mathbb{R}^{2} \cup\{\infty\}$ is the same as the isotopy class of $\Gamma$. By Theorem $5, F_{t}^{l}\left(\Gamma^{\prime}\right)$ does not depend on the left cutting presentations of $\Gamma^{\prime}$. Let $e$ be the edge of $\Gamma^{\prime}$ that is cut to form $T$. Let $C$ and $C^{\prime}$ be the two connected components of $S^{2} \backslash \Gamma$ located on the two sides of $e$, where $C$ is the distinguished component containing $\infty$. Then $\Gamma^{\prime}=\operatorname{cl}_{l}(T)$ and $\Gamma^{\prime \prime}=\operatorname{cl}_{r}(T)=\operatorname{cl}_{l}\left(T^{*}\right)$ are isotopic in $S^{2}$. However $C^{\prime}$ is the distinguished component of $\Gamma^{\prime \prime}$ containing $\infty$. Hence, $\Gamma^{\prime \prime}$ is obtained from $\Gamma^{\prime}$ by a move that consist in pushing the point $\infty$ across 
an edge. The class of $\Gamma^{\prime}$ modulo these moves clearly depends only of $\Gamma$ and thus it is enough to show that $F_{\mathrm{t}}^{l}\left(\Gamma^{\prime}\right)$ is invariant by this move. This is true because

$$
F_{t}^{l}\left(\Gamma^{\prime}\right)=\mathrm{t}_{V^{\varepsilon}}(F(T))=\mathrm{t}_{V^{\varepsilon}}^{\vee}(F(T))=\mathrm{t}_{\left(V^{\varepsilon}\right)^{*}}\left(F(T)^{*}\right)=\mathrm{t}_{V^{-\varepsilon}}\left(F\left(T^{*}\right)\right)=F_{t}^{l}\left(\Gamma^{\prime \prime}\right)
$$

where the second equality is due to $t^{\vee}=t$ on $A$ and the fourth equality follows from (2) and (8).

The invariant $F_{t}^{a}$ of Theorem 6 is a generalization of the analogous invariant defined from ribbon categories in [6]. Moreover, $F_{\mathrm{t}}^{a}$ produces the data of a trivalentambidextrous pair as required in [7]. More precisely, let $B$ be a class of simple objects of $\mathcal{C}$ such that $V^{*} \in \mathrm{B}$ for all $V \in \mathrm{B}$. Denote by $\mathcal{T}_{\mathrm{B}}$ the class of connected trivalent spherical B-colored ribbon graphs (a ribbon graph is trivalent if all its coupons are adjacent to 3 half-edges). Let $\mathrm{d}: \mathrm{B} \rightarrow \mathbb{k}$ be a map such that, for all $V, V^{\prime} \in \mathrm{B}$,

(i) $\mathrm{d}(V)=\mathrm{d}\left(V^{*}\right)$,

(ii) $\mathrm{d}(V)=\mathrm{d}\left(V^{\prime}\right)$ if $V$ is isomorphic to $V^{\prime}$.

For any 1-1-ribbon graph $Q$ with section $(V, \varepsilon)$ where $V$ is simple, we let $\langle Q\rangle=$ $\langle F(Q)\rangle_{V^{\varepsilon}} \in \mathbb{k}$, that is, $F(Q)=\langle Q\rangle \mathrm{id}_{V^{\varepsilon}}$ (see Section 1.6). Using this notation, the pair $(\mathrm{B}, \mathrm{d})$ is trivalent-ambidextrous if for any $\Gamma \in \mathcal{T}_{\mathrm{B}}$ and for any two cutting presentations $T, T^{\prime}$ of $\Gamma$ with sections $(V, \varepsilon)$ and $\left(V^{\prime}, \varepsilon^{\prime}\right)$, we have

$$
\mathrm{d}(V)\langle T\rangle=\mathrm{d}\left(V^{\prime}\right)\left\langle T^{\prime}\right\rangle .
$$

For a trivalent-ambidextrous pair (B, d), we define a function $G_{(\mathrm{B}, d)}: \mathcal{T}_{\mathrm{B}} \rightarrow \mathbb{k}$ by

$$
G_{(\mathrm{B}, d)}(\Gamma)=\mathrm{d}(V)\langle T\rangle
$$

where $T$ is any cutting presentation of $\Gamma$ with section $(V, \varepsilon)$ with $V \in \mathrm{B}$. The definition of a trivalent-ambidextrous pair implies that $G_{(\mathrm{B}, d)}$ is well-defined.

Let us explain how to produce a trivalent-ambidextrous pairs from traces on an ideals and how the invariants derived from such data are related. Let t be a left (resp. right) trace on a left (resp. right) ideal $\mathcal{I}$ of $\mathcal{C}$ and $\mathrm{A}$ as above. Denote by $\mathrm{d}$ the left (resp. right) modified dimension on $\mathcal{I}$ associated with t. Set

$$
\mathrm{B}=\left\{V \in \mathcal{I} \cap \mathcal{I}^{*}: V \text { is simple and } \mathrm{d}(V)=\mathrm{d}\left(V^{*}\right)\right\} .
$$

Corollary 7. The pair $(\mathrm{B}, \mathrm{d})$ is trivalent-ambidextrous, $\mathrm{B} \subset \mathrm{A}$ (and so any $\Gamma \in \mathcal{T}_{\mathrm{B}}$ is $\mathrm{A}$-colored $)$, and $G_{(\mathrm{B}, \mathrm{d})}(\Gamma)=F_{\mathrm{t}}^{a}(\Gamma)$ for all $\Gamma \in \mathcal{T}_{\mathrm{B}}$.

Proof. Clearly $(\mathrm{A}, \mathrm{d})$ is a trivalent-ambidextrous pair since isomorphic objects have equal modified dimension. Now let $f \in \operatorname{End}_{e}(V)$ where $V \in \mathrm{A}$. Since $V$ is simple, we have $f=\langle f\rangle_{V} \operatorname{id}_{V}$ and $\left\langle f^{*}\right\rangle_{V^{*}}=\langle f\rangle_{V}$. Therefore

$$
\mathrm{t}_{V}(f)=\langle f\rangle_{V} \mathrm{~d}(V)=\left\langle f^{*}\right\rangle_{V^{*}} \mathrm{~d}\left(V^{*}\right)=\mathrm{t}_{V^{*}}\left(f^{*}\right)=\mathrm{t}_{V}^{\vee}(f) .
$$


Finally, let $\Gamma \in \mathcal{T}_{\mathrm{B}}$ and $T$ be any cutting presentation of $\Gamma$ with section $(V, \varepsilon)$. Since $V$ is simple, $F(T)=\langle T\rangle \operatorname{id}_{V^{\varepsilon}}$. Then

$$
\begin{aligned}
F_{\mathrm{t}}^{a}(\Gamma) & =\mathrm{t}_{V^{\varepsilon}}(F(T))=\mathrm{t}_{V^{\varepsilon}}\left(\langle T\rangle \mathrm{id}_{V^{\varepsilon}}\right) \\
& =\langle T\rangle \mathrm{t}_{V^{\varepsilon}}\left(\operatorname{id}_{V^{\varepsilon}}\right)=\langle T\rangle \mathrm{d}\left(V^{\varepsilon}\right)=\langle T\rangle \mathrm{d}(V)=G_{(\mathrm{B}, \mathrm{d})}(\Gamma) .
\end{aligned}
$$

Therefore, $\left(F_{t}^{a}\right)_{\mid \mathcal{T}_{\mathrm{B}}}=G_{(\mathrm{B}, \mathrm{d})}$.

\section{Traces and ideals from classes of objects}

In this section we give a systematic approach to defining traces on ideals. In particular, we introduce the notions of a one-sided ambidextrous trace on a class of objects and of a spherical trace on a class of objects. Then we show that such traces are in one to one correspondence with the traces of Section 2.

4.1. Ideals generated by a class of objects. Let $\mathcal{C}$ be a monoidal category. Given a class $\mathcal{O} \subset \mathcal{C}$, set

$$
\begin{aligned}
& \mathcal{I}_{\mathcal{O}}^{l}=\{U \in \mathcal{C}: U \text { is a retract of } Y \otimes X \text { for some } X \in \mathcal{O} \text { and } Y \in \mathscr{C}\}, \\
& \mathcal{I}_{\mathcal{O}}^{r}=\{U \in \mathcal{C}: U \text { is a retract of } X \otimes Z \text { for some } X \in \mathcal{O} \text { and } Z \in \mathcal{C}\}, \\
& \mathcal{I}_{\mathcal{O}}=\{U \in \mathcal{C}: U \text { is a retract of } Y \otimes X \otimes Z \text { for some } X \in \mathcal{O} \text { and } Y, Z \in \mathcal{C}\} .
\end{aligned}
$$

Then $\mathcal{I}_{\mathcal{O}}^{l}$ is the smallest left ideal of $\mathcal{C}$ containing $\mathcal{O}, \mathcal{I}_{\mathcal{O}}^{r}$ is the smallest right ideal of $\mathcal{C}$ containing $\mathcal{O}$, and $\mathcal{I}_{\mathcal{O}}$ is the smallest ideal of $\mathcal{C}$ containing $\mathcal{O}$.

In the case where $\mathcal{O}=\{V\}$ for $V \in \mathcal{C}$, we denote $\mathcal{I}_{\{V\}}^{l}, \mathcal{I}_{\{V\}}^{r}$, and $\mathcal{I}_{\{V\}}$ by $\mathcal{I}_{V}^{l}$, $\mathcal{I}_{V}^{r}$, and $\mathcal{I}_{V}$ respectively.

Lemma 8. Let $\mathcal{C}$ be a pivotal category, $\mathcal{O} \subset \mathcal{C}$, and $U \in \mathcal{C}$.

(a) $U \in \mathcal{I}_{\mathcal{O}}^{l}$ if and only if there exist $X \in \mathcal{O}$ and $f \in \operatorname{End}_{\mathcal{C}}\left(U \otimes X^{*}\right)$ such that

$$
\operatorname{tr}_{r}^{X^{*}}(f)=\mathrm{id}_{U}
$$

(b) $U \in \mathcal{I}_{\mathcal{O}}^{r}$ if and only if there exist $Y \in \mathcal{O}$ and $f \in \operatorname{Ende}_{e}\left(Y^{*} \otimes U\right)$ such that

$$
\operatorname{tr}_{l}^{Y^{*}}(f)=\mathrm{id}_{U}
$$

(c) Let $\mathcal{O}^{\prime}=\left\{X^{*}: X \in \mathcal{O}\right\}$. Then

$$
\left(\mathcal{I}_{\mathcal{O}}^{l}\right)^{*}=\mathcal{I}_{\mathcal{O}^{\prime}}^{r}, \quad\left(\mathcal{I}_{\mathcal{O}}^{r}\right)^{*}=\mathcal{I}_{\mathcal{O}^{\prime}}^{l}, \quad \text { and } \quad I_{\mathcal{O}}=\mathcal{I}_{\mathcal{O}^{\prime}}
$$


Proof. Let us prove (a). Assume $U \in \mathcal{I}_{\mathcal{O}}^{l}$, that is, there exist $X \in \mathcal{O}, Y \in \mathcal{C}$, $p: Y \otimes X \rightarrow U$, and $q: U \rightarrow Y \otimes X$ such that $p q=\mathrm{id}_{U}$. Set

$$
f=\left(p \otimes \operatorname{id}_{X^{*}}\right)\left(\operatorname{id}_{Y} \otimes \widetilde{\operatorname{coev}_{X}} \operatorname{ev}_{X}\right)\left(q \otimes \operatorname{id}_{X^{*}}\right): U \otimes X^{*} \longrightarrow U \otimes X^{*} .
$$

Then $\operatorname{tr}_{r}^{X^{*}}(f)=p q=\mathrm{id}_{U}$. Conversely, assume that there exist $X \in \mathcal{O}$ and $f \in \operatorname{End}_{e}\left(U \otimes X^{*}\right)$ such that $\operatorname{tr}_{r}^{X^{*}}(f)=\operatorname{id}_{U}$. Note that $U \otimes X^{*} \otimes X \in \mathcal{I}_{\mathcal{O}}^{l}$ and set

$$
p=\operatorname{id}_{U} \otimes \operatorname{ev}_{X}: U \otimes X^{*} \otimes X \longrightarrow U
$$

and

$$
q=\left(f \otimes \operatorname{id}_{X}\right)\left(\operatorname{id}_{U} \otimes \widetilde{\operatorname{cov}}_{X}\right): U \longrightarrow U \otimes X^{*} \otimes X .
$$

Then $p q=\operatorname{tr}_{r}^{X^{*}}(f)=\mathrm{id}_{U}$.

One deduces (b) from (a) using $\ell^{\text {rev }}$. The first two identities of (c) follow from the observations in the proof of Lemma 2. Finally $\mathcal{I}_{\mathcal{O}}=\left(\mathcal{I}_{\mathcal{O}}\right)^{*}=I_{\mathcal{O}^{\prime}}$ by Lemma 2 .

4.2. Ambidextrous traces on a class of objects. Let $\ell$ be a pivotal $\mathbb{k}$-category and $\mathcal{O} \subset \mathcal{C}$. Denote by $\varphi=\left\{\varphi_{X}: X \rightarrow X^{* *}\right\}_{X \in \mathcal{C}}$ the pivotal structure of $\mathcal{C}$ (see Section 1.1). Let $t=\left\{t_{X}: \operatorname{End}_{e}(X) \rightarrow \mathbb{k}\right\}_{X \in \mathcal{O}}$ be a family of $\mathbb{k}$-linear forms.

We say that the family $t$ is a left ambidextrous trace on $\mathcal{O}$ if (4) is satisfied for all $X, X^{\prime} \in \mathcal{O}$ and $f \in \operatorname{Ende}_{e}\left(X^{\prime} \otimes X^{*}\right)$, that is

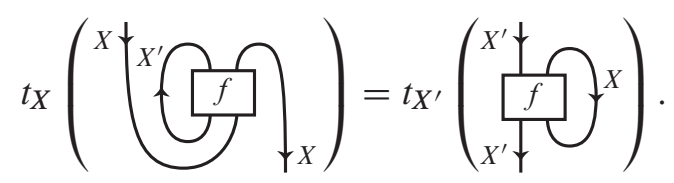

We say that the family $t$ is a right ambidextrous trace on $\mathcal{O}$ if (5) is satisfied for all $X, X^{\prime} \in \mathcal{O}$ and $g \in \operatorname{End}_{\mathcal{C}}\left(X^{*} \otimes X^{\prime}\right)$, that is

$$
t_{X}\left({ }_{x}\left(\Omega^{X^{\prime}}\right)^{\chi^{X}}\right)=t_{X^{\prime}}(x \underbrace{\downarrow^{X^{\prime}}}_{\underbrace{}_{X^{\prime}}}) .
$$

For example, the left (resp. right) trace of endomorphisms in $\mathcal{C}$ (see Section 1.2) is a left (resp. right) ambidextrous trace on $\mathrm{Ob}(\mathcal{C})$.

Remark 9. If $\mathcal{C}$ is a ribbon category and $V \in \mathcal{C}$, we recover the definition of an ambidextrous trace on $\mathcal{O}=\{V\}$ given in [3]. Indeed, in that case, the notions of left and right ambidextrous traces become equivalent: the $\mathbb{k}$-linear map

$$
\gamma: \operatorname{End}_{e}(V \otimes V) \longrightarrow \operatorname{End}_{e}\left(V^{*} \otimes V\right),
$$


defined by

$$
g \longmapsto \gamma(g)=\underbrace{V \uparrow}_{t} \int_{f_{V}}^{t^{V}},
$$

is an isomorphism and, given a map $t_{V}: \operatorname{End}{ }(V) \rightarrow \mathbb{k}$, the morphism $f=\gamma(g)$ satisfies (5) (for $t_{V}$ ) if and only if $t_{V}\left(\operatorname{tr}_{r}^{V}(g)\right)=t_{V}\left(\operatorname{tr}_{l}^{V}(g)\right)$.

By Lemma 4, any left (resp. right) trace on a left (resp. right) ideal $\mathcal{I}$ in $\mathcal{C}$ is a left (resp. right) ambidextrous trace on $\mathcal{I}$ (and in particular on any $\mathcal{O} \subset \mathcal{I}$ ). The following theorem states that one-sided ambidextrous traces on a class of objects bijectively correspond to one-sided traces on the one-sided ideal generated by the class.

Theorem 10. Let $C$ be a pivotal $\mathbb{k}$-category. If $t$ is a left (resp. right) ambidextrous trace on a class $\mathcal{O}$ of objects of $\mathcal{C}$, then there exists a unique left (resp. right) trace $\mathrm{t}$ on $\mathcal{I}_{\mathcal{O}}^{l}\left(\right.$ resp. $\left.\mathcal{I}_{\mathcal{O}}^{r}\right)$ such that $\mathrm{t}_{\mathcal{O}}=t$.

We prove Theorem 10 in Section 4.5.

Corollary 11. Let $\mathcal{C}$ be a pivotal $\mathbb{k}$-category, $\mathcal{I}$ be a left (resp. right) ideal in $\mathcal{C}$, and $t=\left\{t_{X}: \text { Ende }_{\mathcal{C}}(X) \rightarrow \mathbb{k}\right\}_{X \in \mathcal{I}}$ be a family of $\mathbb{k}$-linear maps. Then $t$ is a left (resp. right) trace on $\mathcal{I}$ if and only if $t$ is a left (resp. right) ambidextrous trace on $\mathcal{I}$.

Proof. This is a direct consequence of Theorem 10 and the fact that if $\mathcal{I}$ is a left ideal (resp. right ideal), then $\mathcal{I}_{\mathcal{I}}^{l}=\mathcal{I}\left(\operatorname{resp} . \mathcal{I}_{\mathcal{I}}^{r}=\mathcal{I}\right)$.

4.3. Spherical traces on a class of objects. Let $\ell$ be a pivotal $\mathbb{k}$-category and $t=\left\{t_{X}: \operatorname{End}_{\mathcal{C}}(X) \rightarrow \mathbb{k}\right\}_{X \in \mathcal{I}}$ be a family of $\mathbb{k}$-linear maps.

Lemma 12. The following assertions are equivalent.

(i) The familyt satisfies (6)for all $X, X^{\prime} \in \mathcal{O}, Y \in \mathcal{C}$, and $f \in \operatorname{End} \mathcal{C}\left(X^{\prime} \otimes Y \otimes X^{*}\right)$, that is,

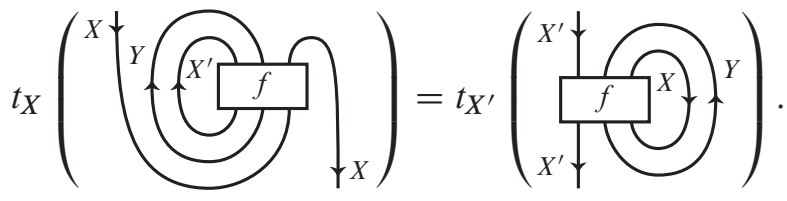

(ii) The familyt satisfies (7) for all $X, X^{\prime} \in \mathcal{O}, Y \in \mathcal{C}$, and $g \in \operatorname{End} \mathcal{C}\left(X^{*} \otimes Y \otimes X^{\prime}\right)$, that is,

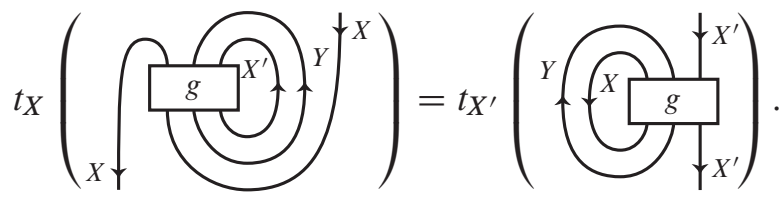


Lemma 12 is proved in Section 4.6.

We say that the family $t$ is a spherical trace on $\mathcal{O}$ if it satisfies the equivalent assertions of Lemma 12. When $\ell$ is spherical (see Section 1.5), the trace of endomorphisms in $\mathcal{C}$ is a spherical trace on $\mathrm{Ob}(\mathcal{C})$.

By Lemma 4, any trace on an ideal $\mathcal{I}$ in $\mathcal{C}$ is an ambidextrous trace on $\mathcal{I}$ (and in particular on any $\mathcal{O} \subset \mathcal{I})$. The following theorem states that spherical traces on a class of objects bijectively correspond to traces on the ideal generated by the class.

Theorem 13. If $t$ is a spherical trace on a class $\mathcal{O}$ of objects of $\mathcal{C}$, then there exists a unique trace $\mathrm{t}$ on $\mathcal{I}_{\mathcal{O}}$ such that $\mathrm{t}_{\mathcal{O}}=t$.

We prove Theorem 13 in Section 4.6.

Corollary 14. Let $\mathcal{I}$ be an ideal in $\mathcal{C}_{\text {and }} t=\left\{t_{X}: \text { Ende }(X) \rightarrow \mathbb{k}\right\}_{X \in \mathcal{I}}$ be a family of $\mathbb{k}$-linear maps. Then $t$ is a trace on $\mathcal{I}$ if and only if $t$ is a spherical trace on $\mathcal{I}$.

Proof. This is a direct consequence of Theorem 13 and the fact that if $\mathcal{I}$ is an ideal, then $\mathcal{I}_{\mathcal{I}}=\mathcal{I}$.

A spherical trace on $\mathcal{O}$ is in particular a left and a right ambidextrous trace on $\mathcal{O}$. But the converse is not true in general (for example, the left trace of endomorphisms in $\mathcal{C}$ is both a left and right ambidextrous trace on $\mathcal{O}=\{\mathbb{1}\}$ but is not a spherical trace

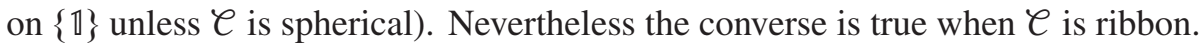

Corollary 15. Assume $\mathcal{C}$ is ribbon. Let $\mathcal{O}$ be a class of objects of $\mathcal{C}$. If $t$ is a right ambidextrous trace on $\mathcal{O}$, then $t$ is a spherical trace on $\mathcal{O}$.

Proof. Since $\mathcal{C}$ is braided, $\mathcal{I}_{\mathcal{O}}^{l}=\mathcal{I}_{\mathcal{O}}^{r}=\mathcal{I}_{\mathcal{O}}$. By Theorem 10,t extends (uniquely) to a right trace t on $\mathcal{I}_{\mathcal{O}}$. Let $X, X^{\prime} \in \mathcal{I}_{\mathcal{O}}$ and $f \in \operatorname{End}_{\mathcal{C}}\left(X^{\prime} \otimes X^{*}\right)$. We have

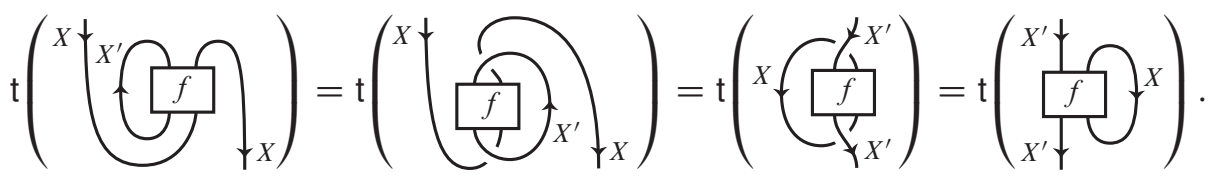

The first and third equalities follow from the fact that $\mathcal{C}$ is ribbon. Since $t$ is a right ambidextrous trace on $\mathcal{I}_{\mathcal{O}}$ by Theorem 10 , then the second equality follows from (5)

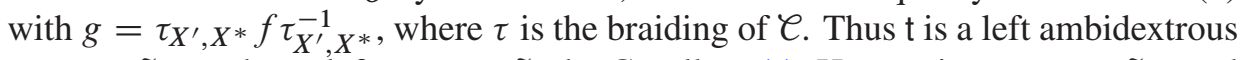
trace on $\mathcal{I}_{\mathcal{O}}$, and so a left trace on $\mathcal{I}_{\mathcal{O}}$ by Corollary 11 . Hence $t$ is a trace on $\mathcal{I}_{\mathcal{O}}$, and so $t=\mathrm{t}_{\mid \mathcal{O}}$ is a spherical trace on $\mathcal{O}$ by Corollary 14 .

4.4. Traces from ambidextrous objects. Let $\varphi$ be a monoidal $\mathbb{k}$-category. If $V$ is a simple object of $\mathcal{C}$, we denote by \langle\rangle$_{V}: \operatorname{End} \mathcal{C}(V) \rightarrow \mathbb{k}$ the inverse of the $\mathbb{k}$-linear isomorphism $\mathbb{k} \rightarrow$ Ende $_{e}(V)$ defined by $k \mapsto k \mathrm{id}_{V}$. 
We say that an object $V$ of $\mathcal{C}$ is left ambidextrous (resp. right ambidextrous, resp. spherical) if it is simple and the $\mathbb{k}$-linear form \langle\rangle$_{V}$ is a left ambidextrous trace (resp. right ambidextrous trace, resp. spherical trace) on $\{V\}$. By Theorems 10 and 13, such an object gives rise to a left trace (resp. right trace, resp. trace) on $\mathcal{I}_{V}^{l}$ (resp. $\mathcal{I}_{V}^{r}$, resp. $\left.\mathcal{I}_{V}\right)$.

The following proposition provides useful characterizations of ambidextrous objects:

Proposition 16. Let $\mathcal{C}$ be a pivotal $\mathbb{k}$-category, with pivotal structure $\varphi$, and let $V$ be a simple object of $\mathcal{C}$.

(a) The following assertions are equivalent.

(i) $V$ is left ambidextrous.

(ii) For all $f \in \operatorname{End}_{e}\left(V^{*} \otimes V\right), \mathrm{ev}_{V} f=\widetilde{\mathrm{ev}}_{V^{*}} f^{*}\left(\operatorname{id}_{V^{*}} \otimes \varphi_{V}\right)$.

(iii) For all $f \in \operatorname{End}_{\varphi}\left(V^{*} \otimes V\right), f \widetilde{\operatorname{coev}_{V}}=\left(\operatorname{id}_{V^{*}} \otimes \varphi_{V}^{-1}\right) f^{*} \operatorname{coev}_{V^{*}}$.

(b) The following assertions are equivalent.

(i) $V$ is right ambidextrous.

(ii) For all $f \in \operatorname{End}_{e}\left(V \otimes V^{*}\right), \widetilde{\mathrm{ev}}_{V} f=\operatorname{ev}_{V^{*}} f^{*}\left(\varphi_{V} \otimes \operatorname{id}_{V^{*}}\right)$.

(iii) For all $f \in \operatorname{End}_{e}\left(V \otimes V^{*}\right), f \operatorname{coev}_{V}=\left(\varphi_{V}^{-1} \otimes \operatorname{id}_{V^{*}}\right) f^{*} \widetilde{\operatorname{cov}}_{V^{*}}$.

(c) $V$ is spherical if and only if

$$
\operatorname{tr}_{r}^{Y}\left(\left(\mathrm{ev}_{V} \otimes \operatorname{id}_{Y}\right) f\right)=\operatorname{tr}_{r}^{Y^{*}}\left(\left(\operatorname{id}_{Y^{*}} \otimes \widetilde{\mathrm{ev}}_{V^{*}}\right) f^{*}\right)\left(\operatorname{id}_{V^{*}} \otimes \varphi_{V}\right)
$$

for all $Y \in \mathcal{C}$ and $f \in \operatorname{Hom}_{\mathcal{C}}\left(Y \otimes V^{*} \otimes V, V^{*} \otimes V \otimes Y\right)$.

Proof. For $Y \in \mathcal{C}$, let $I_{Y}: \operatorname{Hom}_{\mathcal{C}}\left(Y \otimes V^{*} \otimes V, V^{*} \otimes V \otimes Y\right) \rightarrow$ Ende $\left(V \otimes Y \otimes V^{*}\right)$ be the $\mathbb{k}$-linear isomorphism defined by

$$
I_{Y}(f)=\overbrace{V \nmid Y \uparrow V \uparrow} \frac{\downarrow_{V} \downarrow Y}{f} \uparrow^{V} .
$$

Let us prove (a). The form \langle\rangle$_{V}$ is a left ambidextrous trace on $\{V\}$ if and only if (4) applied to $I_{\mathbb{1}}(f)$ is satisfied for all $f \in \operatorname{End}_{\ell}\left(V^{*} \otimes V\right)$. Since $\alpha=\langle\alpha\rangle_{V} \operatorname{id}_{V}$ for all $\alpha \in \operatorname{End}_{e}(V)$, the condition (4) applied to $I_{\mathbb{1}}(f)$ is equivalent to $\operatorname{ev}_{V} f=$ $\widetilde{\mathrm{ev}}_{V^{*}} f^{*}\left(\mathrm{id}_{V^{*}} \otimes \varphi_{V}\right)$. Therefore, (i) is equivalent to (ii). Consequently (i) is equivalent to (iii), since (iii) is (ii) applied to the opposite category $\bigodot^{\text {op }}$ and an object is left ambidextrous in $\mathcal{C}$ if and only if it is left ambidextrous in $\mathcal{C}^{\mathrm{op}}$.

Part (b) is deduced from (a) by using $\ell^{\text {rev }}$.

Let us prove (c). The form \langle\rangle$_{V}$ is a spherical trace on $\{V\}$ if and only if (6) applied to $I_{Y}(f)$ is satisfied for all $Y \in \mathcal{C}$ and $f \in \operatorname{Hom}_{\mathcal{C}}\left(Y \otimes V^{*} \otimes V, V^{*} \otimes V \otimes Y\right)$, which turns out to be equivalent to $\operatorname{tr}_{r}^{Y}\left(\left(\operatorname{ev}_{V} \otimes \operatorname{id}_{Y}\right) f\right)=\operatorname{tr}_{r}^{Y^{*}}\left(\left(\operatorname{id}_{Y^{*}} \otimes \widetilde{\operatorname{ev}}_{V^{*}}\right) f^{*}\right)\left(\operatorname{id}_{V^{*}} \otimes \varphi_{V}\right)$ since $V$ is simple. 
4.5. Proof of Theorem 10. We prove the theorem for left traces. Then the statements for right traces can be deduced using $\ell^{\text {rev }}$. Let $t$ be a left ambidextrous trace on a class $\mathcal{O} \subset \mathcal{C}$. For $U \in \mathcal{I}_{\mathcal{O}}^{l}$ and $\alpha \in \operatorname{End}_{\mathcal{C}}(U)$, set

$$
\mathrm{t}_{U}(\alpha)=t_{X}\left(\operatorname{tr}_{l}^{Y}(q \alpha p)\right)
$$

where $X \in \mathcal{O}, Y \in \mathcal{C}$, and

$$
p: Y \otimes X \longrightarrow U
$$

and

$$
q: U \longrightarrow Y \otimes X
$$

are such that

$$
p q=\mathrm{id}_{U} .
$$

We first verify that $\mathrm{t}_{U}(\alpha)$ does not depend on the choice of $p, q$. Let

$$
p^{\prime}: Y^{\prime} \otimes X^{\prime} \longrightarrow U
$$

and

$$
q^{\prime}: U \longrightarrow Y^{\prime} \otimes X^{\prime},
$$

with $X^{\prime} \in \mathcal{O}, Y^{\prime} \in \mathcal{C}$, such that

$$
p q=\mathrm{id}_{U}
$$

Set

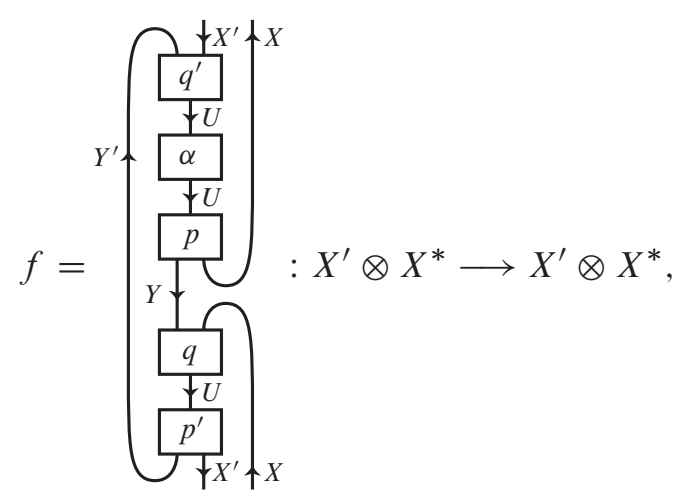

so that

$$
\operatorname{tr}_{r}^{X^{*}}(f)=\operatorname{tr}_{l}^{Y^{\prime}}\left(q^{\prime} \alpha p^{\prime}\right)
$$

and

$$
\varphi_{X}^{-1}\left(\operatorname{tr}_{l}^{X^{\prime}}(f)\right)^{*} \varphi_{X}=\operatorname{tr}_{l}^{Y}(q \alpha p)
$$

Therefore

$$
t_{X^{\prime}}\left(\operatorname{tr}_{l}^{Y^{\prime}}\left(q^{\prime} \alpha p^{\prime}\right)\right)=t_{X^{\prime}}\left(\operatorname{tr}_{r}^{X^{*}}(f)\right)=t_{X}\left(\varphi_{X}^{-1}\left(\operatorname{tr}_{l}^{X^{\prime}}(f)\right)^{*} \varphi_{X}\right)=t_{X}\left(\operatorname{tr}_{l}^{Y}(q \alpha p)\right)
$$


and so $\mathrm{t}_{U}$ is well-defined. Clearly, $\mathrm{t}_{\mathcal{O}}=t$. Let us show that $\mathrm{t}$ is a left trace on $\mathcal{I}_{\mathcal{O}}^{l}$. Let $Z \in \mathcal{C}, U \in \mathcal{I}_{\mathcal{O}}^{l}$, and $\alpha \in \operatorname{End}_{\mathcal{C}}(Z \otimes U)$. Take

$$
p: Y \otimes X \longrightarrow U
$$

and

$$
q: U \longrightarrow Y \otimes X,
$$

with $X \in \mathcal{O}$ and $Y \in \mathcal{C}$, such that

$$
p q=\mathrm{id}_{U} .
$$

Then $\left(\operatorname{id}_{Z} \otimes p\right)\left(\operatorname{id}_{Z} \otimes q\right)=\operatorname{id}_{Z \otimes U}$. Therefore

$\mathrm{t}_{Z \otimes U}(\alpha)=t_{X}\left(\operatorname{tr}_{l}^{Z \otimes Y}\left(\left(\operatorname{id}_{Z} \otimes q\right) \alpha\left(\operatorname{id}_{Z} \otimes p\right)\right)\right)=t_{X}\left(\operatorname{tr}_{l}^{Y}\left(q \operatorname{tr}_{l}^{Z}(\alpha) p\right)\right)=\mathrm{t}_{U}\left(\operatorname{tr}_{l}^{Z}(\alpha)\right)$.

Let now $U, V \in \mathcal{I}_{\mathcal{O}}^{l}, \alpha \in \operatorname{Hom}_{\mathcal{C}}(U, V)$, and $\beta \in \operatorname{Hom}_{\mathcal{C}}(V, U)$. Take

$$
\begin{gathered}
p: Y \otimes X \longrightarrow U, \quad q: U \longrightarrow Y \otimes X, \\
p^{\prime}: Y^{\prime} \otimes X^{\prime} \longrightarrow V, \quad q^{\prime}: V \longrightarrow Y^{\prime} \otimes X^{\prime},
\end{gathered}
$$

with $X, X^{\prime} \in \mathcal{O}$ and $Y, Y^{\prime} \in \mathcal{C}$, such that

$$
p q=\mathrm{id}_{U} \quad \text { and } \quad p^{\prime} q^{\prime}=\mathrm{id}_{V} .
$$

Set

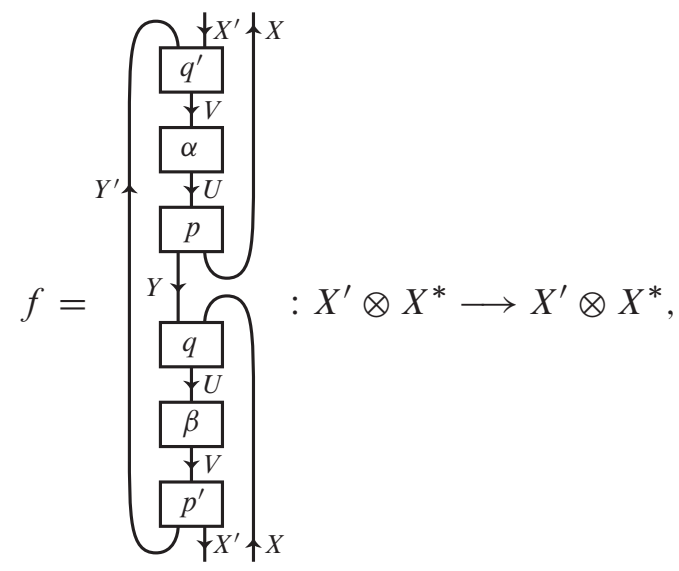

so that

$$
\operatorname{tr}_{r}^{X^{*}}(f)=\operatorname{tr}_{l}^{Y^{\prime}}\left(q^{\prime} \alpha \beta p^{\prime}\right)
$$

and

$$
\varphi_{X}^{-1}\left(\operatorname{tr}_{l}^{X^{\prime}}(f)\right)^{*} \varphi_{X}=\operatorname{tr}_{l}^{Y}(q \beta \alpha p)
$$


Therefore

$$
\begin{aligned}
\mathrm{t}_{V}(\alpha \beta) & =t_{X^{\prime}}\left(\operatorname{tr}_{l}^{Y^{\prime}}\left(q^{\prime} \alpha \beta p^{\prime}\right)\right) \\
& =t_{X^{\prime}}\left(\operatorname{tr}_{r}^{X^{*}}(f)\right) \\
& =t_{X}\left(\varphi_{X}^{-1}\left(\operatorname{tr}_{l}^{X^{\prime}}(f)\right)^{*} \varphi_{X}\right) \\
& =t_{X}\left(\operatorname{tr}_{l}^{Y}(q \beta \alpha p)\right) \\
& =\mathrm{t}_{U}(\beta \alpha) .
\end{aligned}
$$

Hence, $\mathrm{t}$ is a left trace on $\mathcal{I}_{\mathcal{O}}^{l}$. Suppose finally $\ell$ is another left trace on $\mathcal{I}_{\mathcal{O}}^{l}$ with $\ell_{\mid \mathcal{O}}=t$. Let $U \in \mathcal{I}_{\mathcal{O}}^{l}$ and $\alpha \in \operatorname{End}_{\mathcal{C}}(U)$. Take

$$
p: Y \otimes X \longrightarrow U
$$

and

$$
q: U \longrightarrow Y \otimes X
$$

with $X \in \mathcal{O}, Y \in \mathcal{C}$, such that

$$
p q=\mathrm{id}_{U} .
$$

Note that $U$ and $Y \otimes X$ belong to $\mathcal{I}_{\mathcal{O}}^{l}$. Then

$$
\ell_{U}(\alpha)=\ell_{U}(\alpha p q)=\ell_{Y \otimes X}(q \alpha p)=\ell_{X}\left(\operatorname{tr}_{l}^{Y}(q \alpha p)\right)=t_{X}\left(\operatorname{tr}_{l}^{Y}(q \alpha p)\right)=\mathrm{t}_{U}(\alpha) .
$$

Hence, $\ell=\mathrm{t}$.

4.6. Proofs of Lemma 12 and Theorem 13. Let us prove Theorem 13 by taking condition (i) of Lemma 12 as the definition of a spherical trace. Let $t$ be a spherical trace on a class $\mathcal{O} \subset \mathcal{C}$. For $U \in \mathcal{I}_{\mathcal{O}}$ and $\alpha \in \operatorname{End}_{\mathcal{C}}(U)$, set

$$
\mathrm{t}_{U}(\alpha)=t_{X}\left(\operatorname{tr}_{l}^{Y} \operatorname{tr}_{r}^{Z}(q \alpha p)\right)
$$

where $X \in \mathcal{O}, Y, Z \in \mathcal{C}$, and

$$
p: Y \otimes X \otimes Z \longrightarrow U
$$

and

$$
q: U \longrightarrow Y \otimes X \otimes Z
$$

are such that

$$
p q=\mathrm{id}_{U} .
$$

We first verify that $\mathrm{t}_{U}(\alpha)$ does not depend on the choice of $p, q$. Let

$$
p^{\prime}: Y^{\prime} \otimes X^{\prime} \otimes Z^{\prime} \longrightarrow U
$$


and

$$
q^{\prime}: U \longrightarrow Y^{\prime} \otimes X^{\prime} \otimes Z^{\prime},
$$

with $X^{\prime} \in \mathcal{O}$ and $Y^{\prime}, Z^{\prime} \in \mathcal{C}$, such that

$$
p q=\mathrm{id}_{U} .
$$

Set

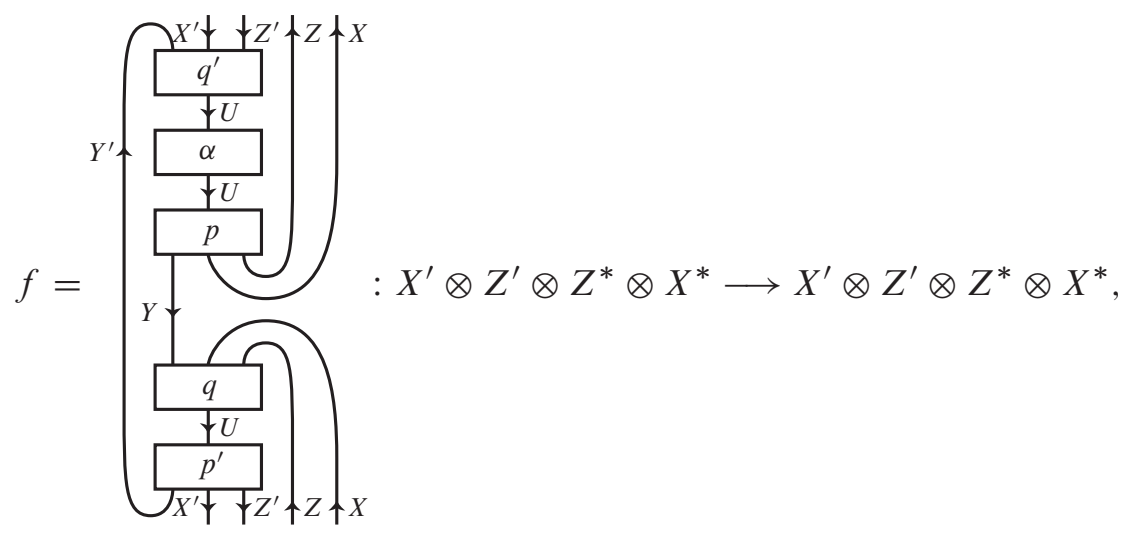

so that

$$
\operatorname{tr}_{r}^{Z^{\prime} \otimes Z^{*} \otimes X^{*}}(f)=\operatorname{tr}_{l}^{Y^{\prime}} \operatorname{tr}_{r}^{Z^{\prime}}\left(q^{\prime} \alpha p^{\prime}\right)
$$

and

$$
\varphi_{X}^{-1}\left(\operatorname{tr}_{l}^{X^{\prime} \otimes Z^{\prime} \otimes Z^{*}}(f)\right)^{*} \varphi_{X}=\operatorname{tr}_{l}^{Y} \operatorname{tr}_{r}^{Z}(q \alpha p) .
$$

Therefore

$$
\begin{aligned}
t_{X^{\prime}}\left(\operatorname{tr}_{l}^{Y^{\prime}} \operatorname{tr}_{r}^{Z^{\prime}}\left(q^{\prime} \alpha p^{\prime}\right)\right) & =t_{X^{\prime}}\left(\operatorname{tr}_{r}^{Z^{\prime} \otimes Z^{*} \otimes X^{*}}(f)\right) \\
& =t_{X}\left(\varphi_{X}^{-1}\left(\operatorname{tr}_{l}^{X^{\prime} \otimes Z^{\prime} \otimes Z^{*}}(f)\right)^{*} \varphi_{X}\right) \\
& =t_{X}\left(\operatorname{tr}_{l}^{Y} \operatorname{tr}_{r}^{Z}(q \alpha p)\right)
\end{aligned}
$$

and so $\mathrm{t}_{U}$ is well-defined. Clearly, $\mathrm{t}_{\mathcal{O}}=t$. Let us show that $\mathrm{t}$ is a trace on $\mathcal{I}_{\mathcal{O}}$. Let $A \in \mathcal{C}, U \in \mathfrak{I}_{\mathcal{O}}, \alpha \in \operatorname{End}_{\mathcal{C}}(A \otimes U)$ and $\beta \in \operatorname{End}_{\mathcal{C}}(U \otimes A)$. Take

$$
p: Y \otimes X \otimes Z \longrightarrow U
$$

and

$$
q: U \longrightarrow Y \otimes X \otimes Z,
$$

with $X \in \mathcal{O}$ and $Y, Z \in \mathcal{C}$, such that

$$
p q=\mathrm{id}_{U} .
$$


Then

$$
\left(\mathrm{id}_{A} \otimes p\right)\left(\mathrm{id}_{A} \otimes q\right)=\mathrm{id}_{A \otimes U}
$$

and

$$
\left(p \otimes \mathrm{id}_{A}\right)\left(q \otimes \mathrm{id}_{A}\right)=\mathrm{id}_{U \otimes A} .
$$

Therefore

$$
\begin{aligned}
\mathrm{t}_{A \otimes U}(\alpha) & =t_{X}\left(\operatorname{tr}_{l}^{A \otimes Y} \operatorname{tr}_{r}^{Z}\left(\left(\operatorname{id}_{A} \otimes q\right) \alpha\left(\operatorname{id}_{A} \otimes p\right)\right)\right) \\
& =t_{X}\left(\operatorname{tr}_{l}^{Y} \operatorname{tr}_{r}^{Z}\left(q \operatorname{tr}_{l}^{A}(\alpha) p\right)\right) \\
& =\mathrm{t}_{U}\left(\operatorname{tr}_{l}^{A}(\alpha)\right)
\end{aligned}
$$

and

$$
\begin{aligned}
\mathrm{t}_{U \otimes A}(\beta) & =t_{X}\left(\operatorname{tr}_{l}^{Y} \operatorname{tr}_{r}^{Z \otimes A}\left(\left(q \otimes \mathrm{id}_{A}\right) \beta\left(p \otimes \mathrm{id}_{A}\right)\right)\right) \\
& =t_{X}\left(\operatorname{tr}_{l}^{Y} \operatorname{tr}_{r}^{Z}\left(q \operatorname{tr}_{r}^{A}(\beta) p\right)\right) \\
& =\mathrm{t}_{U}\left(\operatorname{tr}_{r}^{A}(\beta)\right) .
\end{aligned}
$$

Let now $U, V \in \mathcal{I}_{\mathcal{O}}, \alpha \in \operatorname{Hom}_{\mathcal{C}}(U, V)$, and $\beta \in \operatorname{Hom}_{\mathcal{C}}(V, U)$. Take

$$
p: Y \otimes X \otimes Z \longrightarrow U, \quad q: U \longrightarrow Y \otimes X \otimes Z,
$$

$$
p^{\prime}: Y^{\prime} \otimes X^{\prime} \otimes Z^{\prime} \longrightarrow V, \quad q^{\prime}: V \longrightarrow Y^{\prime} \otimes X^{\prime} \otimes Z^{\prime},
$$

with $X, X^{\prime} \in \mathcal{O}$ and $Y, Y^{\prime}, Z, Z^{\prime} \in \mathcal{\ell}$, such that

$$
p q=\mathrm{id}_{U} \quad \text { and } \quad p^{\prime} q^{\prime}=\mathrm{id}_{V} .
$$

Set

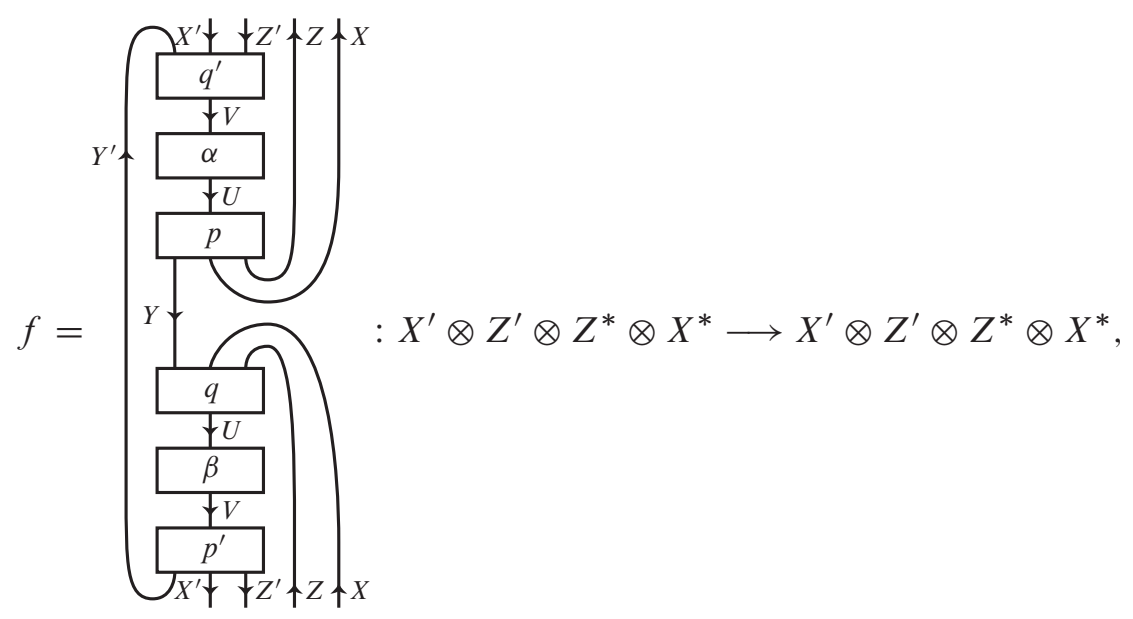


so that

$$
\operatorname{tr}_{r}^{Z^{\prime} \otimes Z^{*} \otimes X^{*}}(f)=\operatorname{tr}_{l}^{Y^{\prime}} \operatorname{tr}_{r}^{Z^{\prime}}\left(q^{\prime} \alpha \beta p^{\prime}\right)
$$

and

$$
\varphi_{X}^{-1}\left(\operatorname{tr}_{l}^{X^{\prime} \otimes Z^{\prime} \otimes Z^{*}}(f)\right)^{*} \varphi_{X}=\operatorname{tr}_{l}^{Y} \operatorname{tr}_{r}^{Z}(q \beta \alpha p)
$$

Therefore

$$
\begin{aligned}
\mathrm{t}_{V}(\alpha \beta) & =t_{X^{\prime}}\left(\operatorname{tr}_{l}^{Y^{\prime}} \operatorname{tr}_{r}^{Z^{\prime}}\left(q^{\prime} \alpha \beta p^{\prime}\right)\right) \\
& =t_{X^{\prime}}\left(\operatorname{tr}_{r}^{Z^{\prime} \otimes Z^{*} \otimes X^{*}}(f)\right) \\
& =t_{X}\left(\varphi_{X}^{-1}\left(\operatorname{tr}_{l}^{X^{\prime} \otimes Z^{\prime} \otimes Z^{*}}(f)\right)^{*} \varphi_{X}\right) \\
& =t_{X}\left(\operatorname{tr}_{l}^{Y} \operatorname{tr}_{r}^{Z}(q \beta \alpha p)\right) \\
& =\mathrm{t}_{U}(\beta \alpha)
\end{aligned}
$$

Hence, $\mathrm{t}$ is a trace on $\mathcal{I}_{\mathcal{O}}$. Suppose finally $\ell$ is another trace on $\mathcal{I}_{\mathcal{O}}$ with $\ell_{\mid \mathcal{O}}=t$. Let $U \in \mathcal{I}_{\mathcal{O}}$ and $\alpha \in \operatorname{End}_{\mathcal{C}}(U)$. Take

$$
p: Y \otimes X \otimes Z \longrightarrow U
$$

and

$$
q: U \longrightarrow Y \otimes X \otimes Z
$$

with $X \in \mathcal{O}$ and $Y, Z \in \mathcal{C}$, such that

$$
p q=\mathrm{id}_{U}
$$

Note that $U$ and $Y \otimes X$ belong to $\mathcal{I}_{\mathcal{O}}$. Then

$$
\begin{aligned}
\ell_{U}(\alpha) & =\ell_{U}(\alpha p q) \\
& =\ell_{Y \otimes X \otimes Z}(q \alpha p) \\
& =\ell_{Y \otimes X}\left(\operatorname{tr}_{r}^{Z}(q \alpha p)\right) \\
& =\ell_{X}\left(\operatorname{tr}_{l}^{Y} \operatorname{tr}_{r}^{Z}(q \alpha p)\right) \\
& =t_{X}\left(\operatorname{tr}_{l}^{Y} \operatorname{tr}_{r}^{Z}(q \alpha p)\right) \\
& =t_{U}(\alpha) .
\end{aligned}
$$

Hence, $\ell=\mathrm{t}$.

Finally, let us prove Lemma 12. Assume the family $t$ satisfies condition (i) of Lemma 12. By the above proof of Theorem 13, there exists a trace $t$ on $\mathcal{I}_{\mathcal{O}}$ such that 
$\mathrm{t}_{\mid \mathcal{O}}=t$ and $\mathrm{t}$ satisfies (7) for all $X, X^{\prime} \in \mathcal{I}_{\mathcal{O}}$ and $Y \in \mathcal{C}$. Since $\mathcal{O} \subset \mathcal{I}_{\mathcal{O}}$ and $\mathrm{t}_{\mid \mathcal{O}}=t$, we have that $t$ satisfies (7) for all $X, X^{\prime} \in \mathcal{O}$ and $Y \in \mathcal{C}$. Hence, condition (i) implies condition (ii). Applying this implication to $\mathcal{C}^{\text {rev }}$ gives the reverse implication, since the ideal generated by $\mathcal{O}$ in $\mathcal{C}^{\text {rev }}$ coincide with the ideal generated by $\mathcal{O}$ in $\mathcal{C}$. This concludes the proof of Lemma 12.

\section{The case of projectives and the slope}

The invariant of Theorem 6 relies on a certain set A of objects defined from a onesided trace on a one-sided ideal. In this section we give a characterization of $A$, in terms of the slope, when the one-sided ideal is the ideal of projective objects.

Let $\mathcal{C}$ be a category. Recall that an object $P$ of $\mathcal{C}$ is projective if the functor $\operatorname{Hom}_{\mathcal{C}}(P,-): \mathcal{C} \rightarrow$ Set preserves epimorphisms, that is, if for any epimorphism $p: X \rightarrow Y$ and any morphism $f: P \rightarrow Y$ in $\mathcal{C}$, there exists a morphism $g: P \rightarrow X$ in $\mathcal{C}$ such that $f=p g$. We denote by $\operatorname{Proj}(\mathcal{C})$ the class of projective objects of $\mathcal{C}$.

An object $Q$ of $\mathcal{C}$ is injective if it is projective in the opposite category $\mathcal{C}^{\mathrm{op}}$. In other words, an object $Q$ of $\mathcal{C}$ is injective if for any monomorphism $i: X \rightarrow Y$ and any morphism $f: X \rightarrow Q$ in $\mathcal{C}$, there exists a morphism $g: Y \rightarrow Q$ in $\mathcal{E}$ such that $f=g i$.

Lemma 17. Let $\mathcal{C}$ be pivotal category.

(a) $\operatorname{Proj}(\mathcal{C})$ is an ideal of $\mathcal{C}$. In particular, $\operatorname{Proj}(\mathcal{C})^{*}=\operatorname{Proj}(\mathcal{C})$.

(b) $\operatorname{Proj}(\mathcal{C})$ is the set of injective objects of $\mathcal{C}$.

(c) If $\mathcal{I}$ is a left (resp. right) ideal of $\mathcal{C}$ containing an object $V$ such that the left evaluation $\mathrm{ev}_{V}: V^{*} \otimes V \rightarrow \mathbb{1}\left(\right.$ resp. the right evaluation $\left.\widetilde{\mathrm{ev}}_{V}: V \otimes V^{*} \rightarrow \mathbb{1}\right)$ is an epimorphism, then $\operatorname{Proj}(\mathcal{C}) \subset \mathcal{I}$.

(d) If $P$ is a projective object such that $\mathrm{ev}_{P}\left(\right.$ resp. $\left.\widetilde{\mathrm{ev}}_{P}\right)$ is an epimorphism, then $\mathcal{I}_{P}^{l}=I_{P}=\operatorname{Proj}(\mathcal{C})\left(\operatorname{resp} . \mathcal{I}_{P}^{r}=\mathfrak{I}_{P}=\operatorname{Proj}(\mathcal{C})\right)$.

Proof. Let us prove (a). Let $P \in \operatorname{Proj}(\mathcal{C}), X \in \mathcal{\ell}, U$ be a retract of $X \otimes P$, and $u: X \otimes P \rightarrow U, v: U \rightarrow X \otimes P$ such that $u v=\operatorname{id}_{U}$. Let $p: M \rightarrow N$ be an epimorphism and $f: U \rightarrow N$ be a morphism. Set

$$
f^{\prime}=\left(\operatorname{id}_{X^{*}} \otimes f u\right)\left(\widetilde{\operatorname{cov}_{X}} \otimes \operatorname{id}_{P}\right): P \longrightarrow X^{*} \otimes N .
$$

Since $\operatorname{id}_{X^{*}} \otimes p$ is an epimorphism, there exists a morphism $g^{\prime}: P \rightarrow X^{*} \otimes M$ such 
that $f^{\prime}=\left(\operatorname{id}_{X^{*}} \otimes p\right) g^{\prime}$. Set $g=\left(\widetilde{\mathrm{ev}}_{X} \otimes \operatorname{id}_{M}\right)\left(\operatorname{id}_{X} \otimes g^{\prime}\right) v: U \longrightarrow M$. Then

$$
\begin{aligned}
p g & =\left(\widetilde{\mathrm{ev}}_{X} \otimes \mathrm{id}_{N}\right)\left(\mathrm{id}_{X} \otimes\left(\mathrm{id}_{X^{*}} \otimes p\right) g^{\prime}\right) v \\
& =\left(\widetilde{\mathrm{ev}}_{X} \otimes \mathrm{id}_{N}\right)\left(\mathrm{id}_{X} \otimes f^{\prime}\right) v \\
& =\left(\widetilde{\mathrm{ev}}_{X} \otimes f\right)\left(\operatorname{id}_{X} \otimes X^{*} \otimes u\right)\left(\mathrm{id}_{X} \otimes \widetilde{\operatorname{cov}}_{X} \otimes \operatorname{id}_{P}\right) v \\
& =f u v \\
& =f .
\end{aligned}
$$

Therefore, $U \in \operatorname{Proj}(\mathcal{C})$, and so $\operatorname{Proj}(\mathcal{C})$ is a left ideal. Likewise one shows that $\operatorname{Proj}(\mathcal{C})$ is a right ideal. Hence, $\operatorname{Proj}(\mathcal{C})$ is an ideal. Thus, Lemma 2 implies that $\operatorname{Proj}(C)^{*}=\operatorname{Proj}(C)$.

Part (b) follows from the fact that the duality functor $\ell^{\text {op }} \rightarrow \zeta$ is an equivalence.

Let us prove the left version of (c), from which the right version can be deduced by using $\ell^{\text {rev }}$. Let $P \in \operatorname{Proj}(\mathcal{C})$. since $\operatorname{id}_{P} \otimes \mathrm{ev}_{V}$ is an epimorphism, there exists a morphism $g: P \rightarrow P \otimes V^{*} \otimes V$ such that $\left(\mathrm{id}_{P} \otimes \mathrm{ev}_{V}\right) g=\operatorname{id}_{P}$. Therefore, $P$ is a retract of $P \otimes V^{*} \otimes V$. Since $P \otimes V^{*} \otimes V \in \mathcal{I}$ as $\mathcal{I}$ is a left ideal, we get $P \in \mathcal{I}$. Hence, $\operatorname{Proj}(\mathcal{C}) \subset \mathcal{I}$.

Finally, let us prove the left version of (d), from which the right version can be deduced by using $\ell^{\text {rev }}$. By (c), we have $\operatorname{Proj}(\mathcal{C}) \subset \mathcal{I}_{P}^{l}$. Now $\mathcal{I}_{P}^{l} \subset \mathcal{I}_{P}$ since $\mathcal{I}_{P}$ is a left ideal containing $P$ and $\mathcal{I}_{P} \subset \operatorname{Proj}(\mathcal{C})$ since $\operatorname{Proj}(\mathcal{C})$ is an ideal containing $P$. Therefore, $I_{P}^{l}=I_{P}=\operatorname{Proj}(\mathcal{C})$.

Now let $\mathcal{C}$ be a pivotal $\mathbb{k}$-category and $P$ be a projective object such that $\mathrm{ev}_{P}$ is an epimorphism. Assume there exists a non zero left ambidextrous trace $t$ on $\{P\}$. For example, such a trace exists when $P$ is left ambidextrous (see Section 4.4). By Lemma $17, \operatorname{Proj}(\mathcal{C})=I_{P}^{l}$ and so, by Theorem 10 , there exists a left trace $t$ on $\operatorname{Proj}(C)$ such that $\mathrm{t}_{P}=t$. Denote by $\mathrm{d}$ the modified left dimension associated with $\mathrm{t}$; see Section 2.2.

Lemma 18. Let $\mathrm{t}$ and $\mathrm{d}$ be the left trace and modified left dimension associated to $P$ and $t$ as defined in the previous paragraph. Let $V$ be a projective object such that $\mathrm{ev}_{V}$ an epimorphism. Then $\mathrm{t}_{V} \neq 0$. Moreover, if $V$ is simple, then $\mathrm{d}(V) \neq 0$.

Proof. Let $g \in \operatorname{End}_{\mathcal{C}}(P)$ such that $\mathrm{t}_{P}(g)=t(g) \neq 0$. By Lemma 17, $\mathfrak{I}_{V}^{l}=$ $\operatorname{Proj}(\mathcal{C})$. By Lemma 8 , since $P \in \mathcal{I}_{V}^{l}$, there exists $f \in \operatorname{End} e\left(P \otimes V^{*}\right)$ such that $\operatorname{id}_{P}=\operatorname{tr}_{r}^{V^{*}}(f)$. Set $h=\varphi_{V}^{-1}\left(\operatorname{tr}_{l}^{P}\left(\left(g \otimes \operatorname{id}_{V^{*}}\right) f\right)\right)^{*} \varphi_{V} \in \operatorname{End}_{e}(V)$. By Theorem 10, $\mathrm{t}$ is a left ambidextrous trace on $\operatorname{Proj}(\mathcal{C})$. Therefore, (4) implies

$$
\mathrm{t}_{V}(h)=\mathrm{t}_{P}\left(\operatorname{tr}_{r}^{V^{*}}\left(\left(g \otimes \mathrm{id}_{V^{*}}\right) f\right)\right)=\mathrm{t}_{P}\left(g \operatorname{tr}_{r}^{V^{*}}(f)\right)=\mathrm{t}_{P}(g) \neq 0 .
$$

Hence, $\mathrm{t}_{V} \neq 0$. Now if $V$ is simple. Let $k \in \mathbb{k}$ such that $h=k \mathrm{id}_{V}$. Then $k \mathrm{~d}(V)=k \mathrm{t}_{V}\left(\operatorname{id}_{V}\right)=\mathrm{t}_{V}(h) \neq 0$, and so $\mathrm{d}(V) \neq 0$. 
Set

$$
\mathrm{A}=\left\{V \in \operatorname{Proj}(\mathcal{C}): V \text { simple and } \mathrm{ev}_{V}, \widetilde{\mathrm{ev}}_{V} \text { are epimorphisms }\right\}
$$

Note that $A^{*}=A$ since the dual of a simple object is simple, $\operatorname{Proj}(C)^{*}=\operatorname{Proj}(C)$, and for any $X \in \mathcal{C}, \mathrm{ev}_{X^{*}}$ is an epimorphism if and only if $\widetilde{\mathrm{ev}}_{X}$ is an epimorphism.

Assume now that $\mathbb{k}$ is a field. For $V \in \mathrm{A}$, the slope of $V$ is

$$
s(V)=\mathrm{d}(V) / \mathrm{d}\left(V^{*}\right) \in \mathbb{k}^{\times} .
$$

The slope is well-defined since $\mathrm{A}^{*}=\mathrm{A}$ and $\mathrm{d}(U) \neq 0$ for all $U \in \mathrm{A}$ (by Lemma 18). Recall $\mathrm{t}^{\vee}$ is the right trace on $\operatorname{Proj}(\mathcal{C})^{*}=\operatorname{Proj}(\mathcal{C})$ defined by $\mathrm{t}_{X}^{\vee}(f)=\mathrm{t}_{X^{*}}\left(f^{*}\right)$ for $X \in \operatorname{Proj}(e)$ and $f \in \operatorname{Ende}_{e}(X)$.

Proposition 19. The slope $s: \mathrm{A} \rightarrow \mathbb{k}^{\times}$has the following properties.

(a) $s\left(V^{*}\right)=s(V)^{-1}$ for all $V \in \mathrm{A}$.

(b) $s(U)=s(V) s(W)$ for all $U, V, W \in \mathrm{A}$ such that $U$ is a retract of $V \otimes W$.

(c) For any $V \in \mathrm{A}, \mathrm{t}_{V}^{\vee}=\mathrm{t}_{V}$ if and only if $s(V)=1$.

Proof. Part (a) is an immediate consequence of the definition since $\mathrm{d}\left(V^{* *}\right)=\mathrm{d}(V)$ because $V^{* *} \simeq V$.

Let us prove (c). Let $f \in \operatorname{Ende}_{e}(V)$. There exists $\lambda \in \mathbb{k}$ such that $f=\lambda \mathrm{id}_{V}$ and so $f^{*}=\lambda \mathrm{id}_{V^{*}}$. Then

$$
\mathrm{t}_{V}(f)=\lambda \mathrm{d}(V)=\lambda \mathrm{d}\left(V^{*}\right) s(V)=s(V) \mathrm{t}_{V^{*}}\left(f^{*}\right)=s(V) \mathrm{t}_{V}^{\vee}(f) .
$$

Hence, $\mathrm{t}_{V}=s(V) \mathrm{t}_{V}^{\vee}$.

Let us prove (b). Let $p \in \operatorname{Hom}_{e}(V \otimes W, U)$ and $q \in \operatorname{Hom}_{e}(U, V \otimes W)$ such that $p q=\mathrm{id}_{U}$. Set

$$
a=\left(p \otimes \operatorname{id}_{W^{*}}\right)\left(\mathrm{id}_{V} \otimes \operatorname{coev}_{W}\right)
$$

and

$$
b=\left(\operatorname{id}_{V} \otimes \widetilde{e v}_{W}\right)\left(q \otimes \operatorname{id}_{W}\right) .
$$

Note that $\operatorname{tr}_{r}^{W^{*}}(a b)=p q=\mathrm{id}_{U}$ and $(b a)^{*}=\operatorname{tr}_{l}^{W^{*}}\left(p^{*} q^{*}\right)$. Since $\mathrm{t}$ is a left trace, 
and in particular satisfies (4) (see Theorem 10), and by using (10), we have

$$
\begin{aligned}
\mathrm{d}(U) & =\mathrm{t}_{U}\left(\operatorname{tr}_{r}^{W^{*}}(a b)\right) \\
& =\mathrm{t}_{W}\left(\varphi_{W}^{-1}\left(\operatorname{tr}_{l}^{U}(a b)\right)^{*} \varphi_{W}\right) \\
& =s(W) \mathrm{t}_{W^{*}}\left(\left(\varphi_{W}^{-1}\left(\operatorname{tr}_{l}^{U}(a b)\right)^{*} \varphi_{W}\right)^{*}\right) \\
& =s(W) \mathrm{t}_{W^{*}}\left(\operatorname{tr}_{l}^{U}(a b)\right) \\
& =s(W) \mathrm{t}_{U \otimes W^{*}}(a b) \\
& =s(W) \mathrm{t}_{V}(b a) \\
& =s(V) s(W) \mathrm{t}_{V^{*}}\left((b a)^{*}\right) \\
& =s(V) s(W) \mathrm{t}_{V^{*}}\left(\operatorname{tr}_{l}^{W^{*}}\left(p^{*} q^{*}\right)\right) \\
& =s(V) s(W) \mathrm{t}_{W^{*} \otimes V^{*}}\left(p^{*} q^{*}\right) \\
& =s(V) s(W) \mathrm{t}_{U^{*}}\left(q^{*} p^{*}\right) \\
& =s(V) s(W) \mathrm{t}_{U^{*}}\left(\mathrm{id}_{U^{*}}\right) \\
& =s(V) s(W) \mathrm{d}\left(U^{*}\right) .
\end{aligned}
$$

Hence, $s(U)=s(V) s(W)$.

Remark that when $\ell$ is ribbon, meaning that $\ell$ is endowed with a braiding $\tau$ such that its associated twist

$$
\theta=\left\{\theta_{X}=\operatorname{tr}_{r}^{X}\left(\tau_{X, X}\right): X \rightarrow X\right\}_{X \in \mathcal{C}}
$$

is self-dual (i.e., $\left(\theta_{X}\right)^{*}=\theta_{X} *$ for all $X \in \mathcal{C}$ ), then $s(V)=1$ for all $V \in \mathrm{A}$ (see [6]), and so $^{\vee}=\mathrm{t}$ on $\mathrm{A}$.

\section{References}

[1] C. De Concini and V. G. Kac, Representations of quantum groups at roots of 1. In A. Connes et al. (eds.), Operator algebras, unitary representations, enveloping algebras, and invariant theory. Papers from the colloquium in honor of Jacques Dixmier held at the Institut Henri Poincaré, Paris, May 22-26, 1989. Progress in Mathematics 92. Birkhäuser Boston, 1990. MR 1103601 Zbl 0738.17008

[2] C. De Concini, C. Procesi, N. Reshetikhin, and M. Rosso, Hopf algebras with trace and representations. Invent. Math. 161 (2005), 1-44. MR 2178656 Zbl 1132.17007

[3] N. Geer, J. Kujawa, and B. Patureau-Mirand, Generalized trace and dimension functions on ribbon categories. Selecta Math. 17 (2011), 453-504. MR 2803849 
[4] N. Geer, J. Kujawao and B. Patureau-Mirand, Ambidextrous objects and trace functions for nonsemisimple categories. To appear in Proc. Amer. Math. Soc. Preprint 2011. arXiv: $1106.4477 \mathrm{v} 3$

[5] N. Geer and B. Patureau-Mirand, Topological invariants from non-restricted quantum groups. Preprint 2010. arXiv:1009.4120v1

[6] N. Geer, B. Patureau-Mirand, and V. Turaev, Modified quantum dimensions and re-normalized link invariants. Compos. Math. 145 (2009), 196-212. MR 2480500 Zbl 1160.81022

[7] N. Geer, B. Patureau-Mirand, and V. Turaev, Modified $6 j$-symbols and 3-manifold invariants. Adv. Math. 228 (2011), 1163-1202. MR 2822220 Zbl 05949102

[8] G. Maltsiniotis, Traces dans les catégories monoïdales, dualité et catégories monoïdales fibrées. Cahiers Topologie Géom. Différentielle Catég. 36 (1995), 195-288. MR 1352535 Zbl 0831.18002

Received March 31, 2011

Nathan Geer, Department of Mathematics and Statistics, Utah State University,

Logan, UT 84322 Logan, U.S.A.

E-mail: nathan.geer@usu.edu

Bertrand Patureau-Mirand, LMBA UMR 6205,

Université Européenne de Bretagne - Université de Bretagne-Sud,

BP 573, 56017 Vannes, France

E-mail: bertrand.patureau@univ-ubs.fr

Alexis Virelizier, Département de mathématiques, Université Montpellier 2,

34095 Montpellier Cedex 5, France

E-mail: virelizi@math.univ-montp2.fr 